Proceedings of the 9th Workshop on Quantum Chaos and Localisation Phenomena, May 24-26, 2019, Warsaw, Poland

\title{
Circular and Elliptical Neutrino Billiards: A Semiclassical Approach
}

\author{
B. DieTz* \\ School of Physical Science and Technology, and Key Laboratory for Magnetism and Magnetic Materials of MOE, \\ Lanzhou University, Lanzhou, Gansu 730000, China
}

\begin{abstract}
Quantum signatures of classical chaos in the spectral properties of non-relativistic quantum billiards are well understood by now based on the semiclassical and the random matrix theory aproach. The experimental detection of relativistic phenomena featured by graphene implicated an increasing interest in the properties of graphene billiards and relativistic quantum billiards consisting of massless spin- $1 / 2$ particles governed by the Dirac equation and confined to a planar domain. Confinement is attained by imposing appropriate boundary conditions on the wave function components. We chose those proposed for neutrino billiards. By now numerical studies have been performed on the spectral properties of neutrino billiards of various shapes. Our main focus is the question to what extent the semiclassical approach in terms of trace formulae applies to such systems. We derive trace formulae for circular and elliptical neutrino billiards. The associated eigenvalue equations have been solved analytically for the circular billiard. We present analytical solutions for elliptical neutrino billiards.
\end{abstract}

DOI: 10.12693/APhysPolA.136.770

PACS/topics: billiards, semiclassical theory, relativistic quantum chaos

\section{Introduction}

A classical billiard [1-4] (CB) is a bounded twodimensional domain, in which a point particle moves freely whereas upon impact with the boundary it is reflected elastically. Since its dynamics only depends on its shape, such systems are widely used to investigate the effects of the classical dynamics on properties of the associated quantum system [5-8], referred to as quantum billiard (QB) in the sequel. The eigenstates of a $\mathrm{QB}$ are obtained by solving the Schrödinger equation of a free particle and imposing the Dirichlet boundary condition on the wave functions, that is, requiring that they should vanish along the boundary. Within the field of quantum chaos numerous numerical and also experimental studies with microwave billiards [9-15] on the quantum signatures of classical chaos focused, e.g., on the validity of the Bohigas-Giannoni-Schmit (BGS) conjecture [16-19] for chaotic systems and the Berry-Tabor (BT) conjecture [20] for integrable ones. The former states that the spectral fluctuation properties of a chaotic system coincide with those of random matrices from the Gaussian orthogonal ensemble (GOE), if time-reversal $\mathcal{T}$ invariance is conserved, and with those of random matrices from the Gaussian unitary ensemble (GUE), if it is violated. According to the BT conjecture the fluctuation properties in the eigenvalue spectra of a quantum system with classically integrable counterpart are well described by those of random Poissonian numbers. Both conjectures were confirmed for typical quantum systems by

*e-mail: dietz@lzu.edu.cn these theoretical, numerical, and experimental studies. Yet, there also exist examples of billiards with certain shapes of which the spectral properties were shown to exhibit GUE statistics even though $\mathcal{T}$ invariance is preserved, that is, to deviate from the expected behavior. One example are billiards of which the shapes have a threefold symmetry and no further one like, e.g., a mirror symmetry [21-23], another one are constant width billiards [24-26]. In these billiards the observed GUE behavior is attributed to the unidirectionality of the associated classical dynamics.

Already in 1987 Berry and Mondragon proposed neutrino billiards [27] (NBs) as another example exhibiting GUE statistics when their shape gives rise to a chaotic classical dynamics and has no geometric symmetries. Neutrino billiards are described by the Dirac equation of massless spin- $1 / 2$ particles which are confined to a bounded two-dimensional domain by imposing the requirement that there is no outward current. Note that these billiards were introduced and given their names before the revelation that neutrinos have mass. It was shown in this reference that NBs with a circular shape exhibit Poissonian statistics whereas the spectral properties of NBs with the shape of a chaotic Africa billiard coincide with those of random matrices from the GUE. Interest in NBs re-emerged with the pioneering fabrication of graphene $[28]$ and the thereby triggered interest in the spectral properties of graphene billiards [29-37], that is, finite size sheets of (artificial) graphene. Since NBs do not have a classical counterpart the question arose, whether their spectral properties depend only on the shape of the billiard as in the corresponding QBs. In order to gain insight into this problem we focus on the semiclassical limit of the eigenvalue problem of circular and elliptical NBs. 
Actually, a second important issue within the field of quantum chaos concerns the semiclassical approach. One of the most important achievements was the periodic orbit theory pioneered by Gutzwiller [38], which rendered possible the understanding of the impact of the classical dynamics on the properties of the quantum system in terms of purely classical quantities. To be explicit, Gutzwiller derived a semiclassical approximation of the fluctuating part of the spectral density in terms of a trace formula, that is, a sum over periodic orbits [39], which is applicable to systems for which all periodic orbits are isolated, that is, basically to classically chaotic ones. The derivation started from a semiclassical approximation of the quantum time-evolution operator, namely the van-Vleck propagator, deduced by Gutzwiller from the Feynman path integral by applying a stationary phase approximation [5]. A few years later an analogous trace formula was obtained for integrable systems by Berry and Tabor [20] based on the semiclassical EinsteinBrillouin-Keller (EBK) quantization [40-42]. A severe drawback of these trace formulae are the convergence problems one generally has to struggle with when summing over the periodic orbits. These may be overcome by formulating the semiclassical quantization condition based on the dynamical $\zeta$ function [43-45] starting from the spectral determinant of which the zeroes yield the eigenvalues of the quantum system. Harayama and Shudo derived such a spectral determinant for twodimensional billiards with a chaotic classical dynamics from a boundary integral equation [46] for the eigenvalues and recovered in the semiclassical limit Gutzwiller's trace formula. They had to deal with determinants of which the dimension coincides with the number of points of impact of the trajectory with the boundary, i.e., with the period of the orbit. In Refs. [47, 48] a procedure for the semiclassical quantization was obtained for chaotic planar and three-dimensional billiards directly from the boundary integral equation, thus avoiding large matrices.

A semiclassical trace formula and semiclassical quantization rules were derived for the massive Dirac equation incorporating the spin dynamics in Refs. [49, 50]. Furthermore, trace formulae were derived for graphene in [51]. In the present article we will derive a trace formula for circular and elliptic NBs by proceeding as in Refs. [47, 48]. In Sect. 2 we will briefly review the relevant results on the eigentstates and trace formulae for circular [52, 53] and elliptical QBs [54]. In Sect. 3 we will briefly review the boundary integral equation for NBs and then in Sect. 4 derive a trace formula for NBs with a smooth boundary, and finally conclude the article in Sect. 5 .

\section{Non-relativistic circular and elliptical QBs}

In this section we briefly recapitulate the features of the eigenstates and the trace formulae of circular and elliptical QBs which are of relevance for the present article.

\subsection{Circular billiard}

The shape of a circular billiard exhibits rotational symmetry of infinite order so that besides the energy the angular momentum is conserved and, accordingly, the classical dynamics is integrable. The eigenfunctions and eigenvalues are obtained by imposing the Dirichlet boundary condition on the solutions of the Schrödinger equation of a free particle in polar coordinates $(x(r, \phi), y(r, \phi))=r(\cos \phi, \sin \phi)$, with the billiard domain $\Omega$ of a circular billiard with radius unity defined by $\Omega=\{(r, \phi): 0 \leq r \leq 1,0 \leq \phi<2 \pi\}$. This yields for the eigenfunctions

$$
\psi_{l, n}(r, \phi)=J_{l}\left(k_{l, n} r\right) g_{l}(\phi),
$$

where $g_{l}(\phi)=\cos (l \phi)$ for solutions which are symmetric with respect to the $x$ axis and $g_{l}(\phi)=\sin (l \phi)$ for the antisymmetric ones and $l=0,1,2, \cdots$ with $l=0$ excluded for the antisymmetric solutions. The eigenvalues $E_{l, n}$ correspond to the squares of the eigenwavenumbers $k_{l, n}$, $E_{l, n}=k_{l, n}^{2}$ which are given by the zeroes of the Bessel function $J_{l}(x)$ :

$$
J_{l}\left(k_{l, n}\right)=0 .
$$

The spectral properties coincide with those of uncorrelated Poissonian random number and thus with those of generic billiards with an integrable classical dynamics, see Fig. 1.

We are interested in the semiclassical limit of relativistic NBs. For this we derive a trace formula which expresses the fluctuating part of the spectral density of a NB in terms of a sum over classical periodic orbits
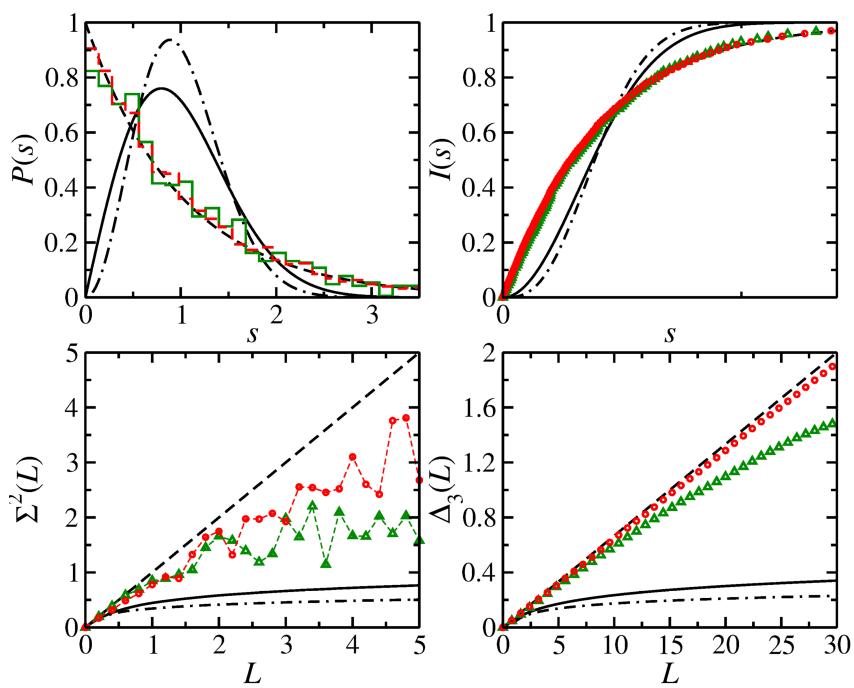

Fig. 1. Spectral properties of the circular QB (green full line and triangles, 1000 levels) and NB (red dashed line and circles, 5000 levels). Shown are the nearestneighbor spacing distribution $P(s)$, the integrated nearest-neighbor spacing distribution $I(s)$, the number variance $\Sigma^{2}(L)$ and the spectral rigidity $\Delta_{3}(L)$ [57]. Note that the deviations from Poisson decrease with increasing level numbers. 


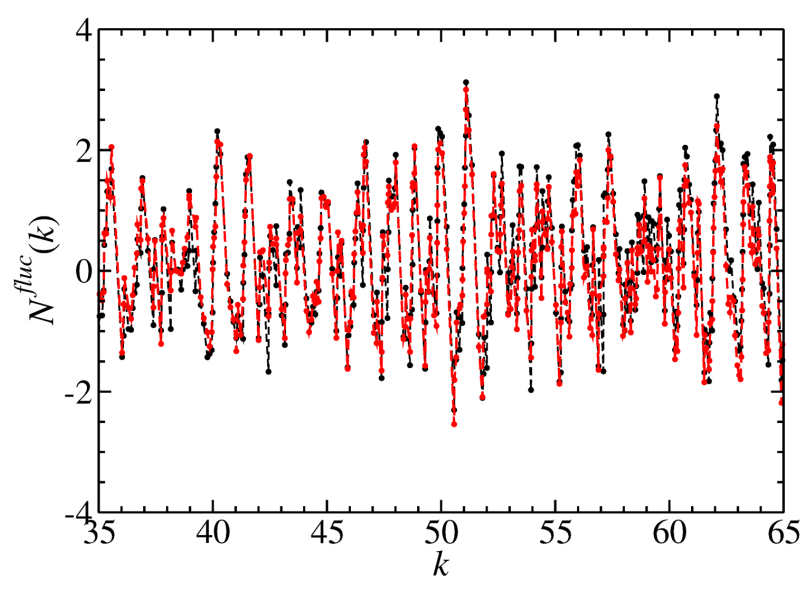

Fig. 2. Comparison of the fluctuating part of the integrated spectral density deduced from the eigenvalues of the circular QB (black dots) with that obtained by integrating the trace formula over $k$ (red (gray) dots). The dots are connected by dashed lines to guide the eye of the reader.

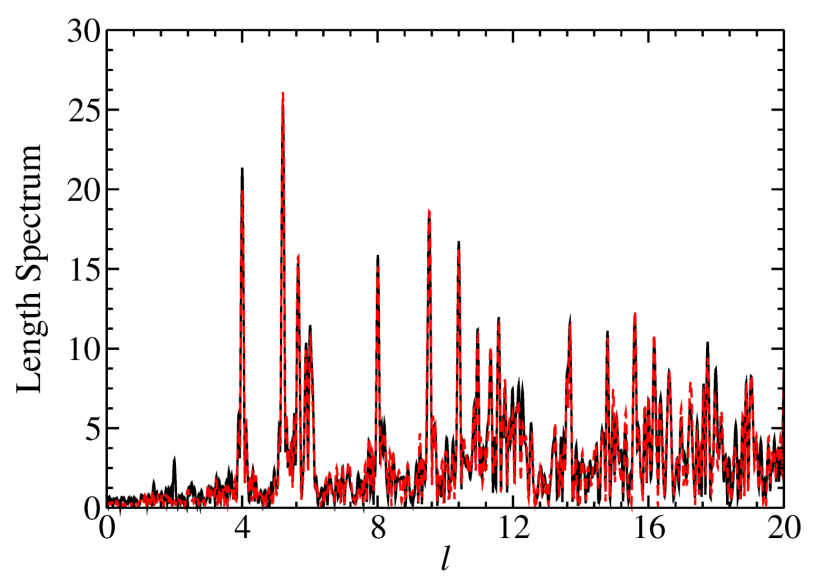

Fig. 3. Comparison of the length spectrum deduced from the fluctuating part of the spectral density of the circular QB (black line) with that obtained from the trace formula (red dashed line).

of the CB. As will be shown below, it is directly related to the trace formula of the corresponding $\mathrm{QB}$, which for the circular QB is given by $[20,52,53]$ :

$$
\begin{aligned}
& \rho(k)=\rho^{\operatorname{smooth}}(k)+\sqrt{\frac{k}{\pi}} \sum_{M} m_{M} \frac{\left(\sin \frac{m_{\varphi}}{p} \pi\right)^{3 / 2}}{\sqrt{p}} \\
& \quad \times \cos \left(2 k p \sin \left(\frac{m_{\varphi}}{p} \pi\right)-\frac{3 \pi}{2} p+\frac{\pi}{4}\right),
\end{aligned}
$$

where $\boldsymbol{M}=\left(p, m_{\varphi}\right)$ with $p$ and $m_{\varphi}$ denoting the number of bounces at the billiard boundary and of turns around the center, respectively, and

$$
m_{M}= \begin{cases}1, & p=2 m_{\varphi} \\ 2 & , p>2 m_{\varphi} \\ 0, & \text { otherwise }\end{cases}
$$

The smooth part of the spectral density, $\rho^{\text {smooth }}(k)$ is given by Weyl's formula [56]:

$$
\rho^{\text {Weyl }}(k)=\frac{\mathcal{A}}{2 \pi} k-\frac{\mathcal{L}}{4 \pi} .
$$

Note that due to the rapid increase of the number of periodic orbits with increasing action $s_{p}=2 p \sin \left(\frac{m_{\varphi}}{p} \pi\right)$ and due to the occurrence of accumulation points for fixed $m_{\varphi}$ and increasing $p$, where the total lengths $s_{p}$ of the periodic orbits approach a multiple of $2 \pi$, the trace formula, given by the sum in Eq. (3) does not converge. In Figs. 2 and 3 we compare the fluctuating part of the integrated spectral density $N(k)$ and the length spectrum, that is the modulus of the Fourier transform of the fluctuating part of $\rho(k)$ deduced from the eigenvalues of the circular QB with those computed from the trace formula, respectively. The lengths spectra exhibit peaks at the lengths of the periodic orbits.

\subsection{Elliptical billiard}

The classical dynamics of a particle in an elliptical billiard is also integrable because it has a second constant of motion, which is the product of the angular momenta $L_{1}$ and $L_{2}$ with respect to the two focal points. In terms of elliptical coordinates,

$$
\begin{aligned}
& (x(\mu, \phi), y(\mu, \phi))=(\cosh \mu \cos \phi, \sinh \mu \sin \phi), \\
& \Omega=\left\{(\mu, \phi): 0 \leq \mu \leq \mu_{0}, 0 \leq \phi<2 \pi\right\},
\end{aligned}
$$

with $\mu=\mu_{0}$ defining the boundary of an ellipse with semiminor axis $b=\sinh \mu_{0}$ and semimajor axis $a=\cosh \mu_{0}$, it is given by

$$
L_{1} L_{2}=\frac{p_{\phi}^{2} \sinh ^{2} \phi-p_{\mu}^{2} \sin ^{2} \phi}{\cosh ^{2} \mu-\cos ^{2} \phi} .
$$

Here, $p_{\mu}$ and $p_{\phi}$ denote the canonical momenta

$$
\begin{aligned}
p_{\mu} & =\frac{1}{2} \dot{\mu}\left(\cosh ^{2} \mu-\cos ^{2} \phi\right), \\
p_{\phi} & =\frac{1}{2} \dot{\phi}\left(\cosh ^{2} \mu-\cos ^{2} \phi\right) .
\end{aligned}
$$

The ellipse has eccentricity $\epsilon=\frac{1}{\cosh \mu_{0}}$, and the area and perimeter equal $\mathcal{A}=\pi \cosh \mu_{0} \sinh \mu_{0}$ and $\mathcal{L}=4 E(\epsilon) \cosh \mu_{0}$, respectively, where $E(\epsilon)=E\left(\frac{\pi}{2} ; \epsilon\right)$ is the complete elliptic integral of the second kind with

$$
E(\phi ; \lambda)=\int_{0}^{\phi} \mathrm{d} \theta \sqrt{1-\lambda^{2} \sin ^{2} \theta} .
$$

The eigenfunctions and eigenvalues are obtained by writing the Schrödinger equation in terms of elliptical coordinates yielding two separate equations for $\phi$ and $\mu$, which, in distinction to the case of the circular billiard, are linked by a common constant $h=h(k)$, which depends on $k$ :

$$
\begin{aligned}
& \frac{\partial^{2}}{\partial \phi^{2}} \Phi(\phi)+\left(h(k)-\frac{k^{2}}{2}-\frac{k^{2}}{2} \cos 2 \phi\right) \Phi(\phi)=0, \\
& -\frac{\partial^{2}}{\partial \mu^{2}} M(\mu)+\left(h(k)-\frac{k^{2}}{2}-\frac{k^{2}}{2} \cosh 2 \mu\right) M(\mu)=0 .
\end{aligned}
$$



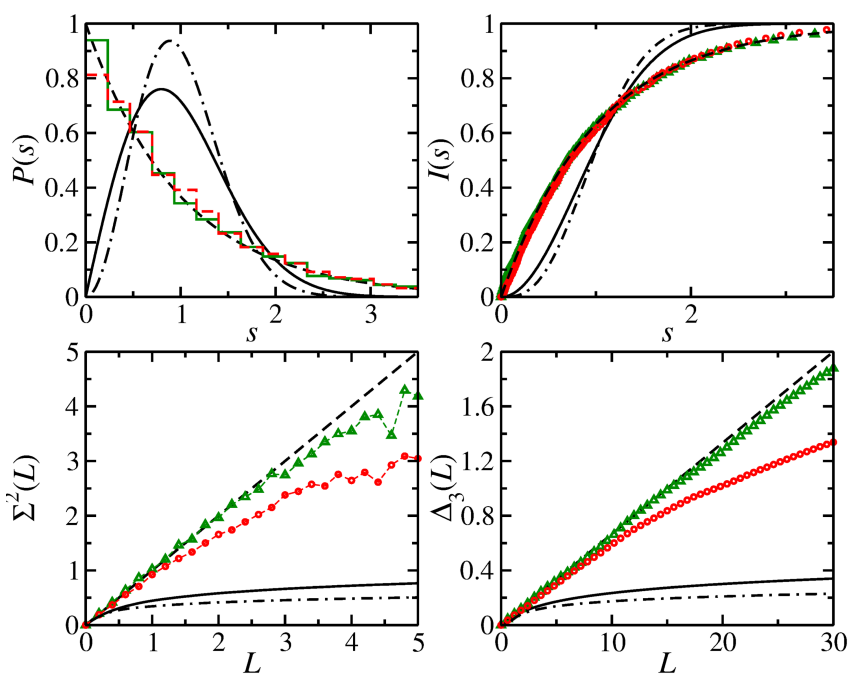

Fig. 4. The same as in Fig. 1 for the quarter ellipse QB (green full line and triangles, 2000 levels) and NB (red dashed line and circles, 1000 levels).

The solutions correspond to products of the radial and the periodic Mathieu functions [57-61]:

$$
\psi(k ; \mu, \phi) \propto M_{l}(k ; \mu) \Phi_{l}(k ; \phi),
$$

see also Appendix for further information on the representation of the Mathieu functions used in the present article. The eigenvalues are obtained by imposing Dirichlet boundary conditions on the wave functions and, accordingly, correspond to the zeroes of the radial Mathieu functions,

$$
M_{l}\left(k_{l, n} ; \mu_{0}\right)=0 .
$$

The periodic Mathieu functions $\Phi_{n}(\phi)$ may be classified according to their reflection symmetry with respect to the major and minor axes of the elliptical billiard. The spectral properties of the ellipse QB again coincide with those of uncorrelated Poissonian random number, see Fig. 4 (for all presented results we chose $\mu_{0}=1$ ). The spectral properties for the fully desymmetrized elliptical QB correspond to a quarter ellipse billiard with the Dirichlet boundary conditions. Note that the half and quarter ellipse are the only ellipse sector billiards with integrable dynamics [54], whereas all other sectors exhibit mixed dynamics.

For the elliptical billiard the derivation and also the final result for the trace formula are more complex than for the circular one [54]. In the ellipse two types of motions occur, depending on the sign of the energy-independent quantity $\gamma=\frac{L_{1} L_{2}}{k^{2}}$ with $-1 \leq \gamma \leq b^{2}$. For $-1 \leq \gamma<0$ the trajectories always cross the $x$-axis between the two focal points and are confined to the domain enclosed by the upper and lower ellipse boundaries and two hyperbolas with semiconjugate axes $b^{\prime}= \pm \sqrt{-\gamma}$, whereas for $\gamma>0$ it is similar to the rotational motion in a circular billiard and motion is confined to the region between the billiard boundary and an inner ellipse with semiminor axis $b^{\prime}=\sqrt{\gamma}$.

For the case $\gamma \leq 0$ the periodic orbits are obtained from the condition

$F\left(\arcsin \sqrt{\frac{b^{2}}{b^{2}-\gamma_{p, m_{\phi}}^{h}}} ; \frac{1}{\kappa_{p, m_{\varphi}}^{h}}\right)=\frac{m_{\varphi}}{p} F\left(\frac{\pi}{2} ; \frac{1}{\kappa_{p, m_{\varphi}}^{h}}\right)$,

with $p=2,3, \cdots$ and $1 \leq m_{\varphi} \leq p$ and the number of reflections and rotations about the center of the ellipse equal to $2 p$ and $m_{\varphi}$, respectively. Furthermore,

$$
F(\phi ; \lambda)=\int_{0}^{\phi} \frac{\mathrm{d} \theta}{\sqrt{1-\lambda^{2} \sin ^{2} \theta}}
$$

denotes the elliptical integral of the second kind and $\kappa=\frac{1}{\sqrt{1+\gamma}}$. The lengths of these periodic orbits are given by

$$
\begin{aligned}
& l_{p, m_{\varphi}}^{h}=\frac{4 p a b}{\sqrt{b^{2}-\gamma_{p, m_{\varphi}}^{h}}}-4 p E\left(\sqrt{\frac{b^{2}}{b^{2}-\gamma_{p, m_{\varphi}}^{h}}}, \frac{1}{\kappa_{p, m_{\varphi}}^{h}}\right) \\
& \quad+4 m_{\varphi} E\left(\frac{\pi}{2} ; \frac{1}{\kappa_{p, m_{\varphi}}^{h}}\right) .
\end{aligned}
$$

For the case $\gamma>0$ the periodic orbits are determined from the condition

$$
F\left(\arcsin \sqrt{\frac{b^{2}-\gamma_{p, m_{\varphi}}^{e}}{b^{2}}} ; \kappa_{p, m_{\varphi}}^{e}\right)=\frac{2 m_{\varphi}}{p} F\left(\frac{\pi}{2} ; \kappa_{p, m_{\varphi}}^{e}\right),
$$

and their lengths are given by

$$
\begin{aligned}
& l_{p, m_{\varphi}}^{e}=\frac{2 p a}{b} \sqrt{b^{2}-\gamma_{p, m_{\varphi}}^{e}} \\
& -\frac{2 p}{\kappa_{p, m_{\varphi}}^{e}} E\left(\sqrt{\frac{b^{2}-\gamma_{p, m_{\varphi}}^{e}}{b^{2}}}, \kappa_{p, m_{\varphi}}^{e}\right) \\
& +\frac{4 m_{\varphi}}{\kappa_{p, m_{\varphi}}^{e}} E\left(\frac{\pi}{2} ; \kappa_{p, m_{\varphi}}^{e}\right)
\end{aligned}
$$

for an orbit with $p=3,4, \ldots$ reflections and $1 \leq m_{\varphi}<p / 2$ rotations around the center of the ellipse. The trace formula for the elliptical billiard was derived in Refs. [54,61]. We used the trace formula in the form provided by Sieber, yielding

$$
\rho(k)=\rho^{\text {smooth }}(k)+\sum_{p=3}^{\infty} \sum_{m_{\varphi}=1}^{\left[\frac{p-1}{2}\right]} \sqrt{\frac{2 k \gamma_{p, m_{\varphi}}^{e}}{\pi}} \frac{4 l_{p, m_{\varphi}}^{e} F\left(\frac{\pi}{2}, \kappa_{p, m_{\varphi}}^{e}\right)}{p \pi \sqrt{\frac{2 p a b}{\sqrt{b^{2}-\gamma_{p, m_{\varphi}}^{e}}}-l_{p, m_{\varphi}}^{e}}} \cos \left(k l_{p, m_{\varphi}}^{e}-p \frac{3 \pi}{2}+\frac{\pi}{4}\right)+
$$




$$
\begin{aligned}
& +\sum_{p=2}^{\infty} \sum_{m_{\varphi}=1}^{p-1} \sqrt{\frac{2 k\left|\gamma_{p, m_{\varphi}}^{h}\right|}{\pi}} \frac{4 l_{p, m_{\varphi}}^{h} F\left(\frac{\pi}{2}, \frac{1}{\kappa_{p, m_{\varphi}}^{h}}\right)}{2 p \pi \kappa_{p, m_{\varphi}}^{h} \sqrt{\frac{4 p a b}{\sqrt{b^{2}-\gamma_{p, m_{\varphi}}^{h}}}-l_{p, m_{\varphi}}^{h}}} \\
& \times\left[\cos \left(k l_{p, m_{\varphi}}^{h}-m_{\varphi} \pi-\frac{\pi}{4}\right)+\frac{1}{\sqrt{2 \pi}} \int_{\Lambda}^{\infty} \mathrm{d} x \frac{\sin \left(k l_{p, m_{\varphi}}^{h}-m_{\varphi} \pi-\frac{x^{2}}{2}\right)}{x^{2}}\right] \\
& +\sum_{m_{\varphi}=1}^{\infty} \frac{4 b}{\pi} \frac{\sin \left(4 m_{\varphi} k b\right)}{2 \sin \left(2 m_{\varphi} \arcsin \frac{b}{a}\right)}+\sum_{m_{\varphi}=1}^{\infty} \frac{4 a}{\pi} \frac{\cos \left(4 m_{\varphi} k a-m_{\varphi} \pi\right)}{2 \sinh \left(2 m_{\varphi} \arcsin \frac{1}{b}\right)}
\end{aligned}
$$

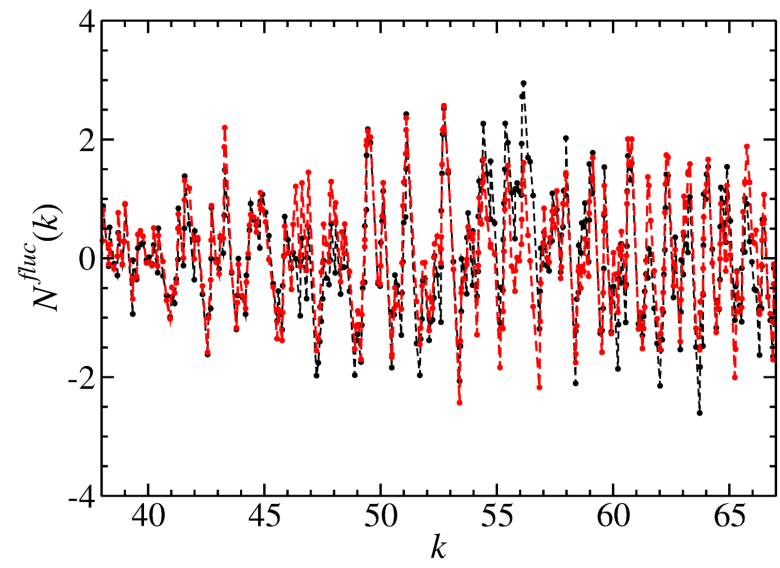

Fig. 5. The same as Fig. 2 for the quarter ellipse QB.

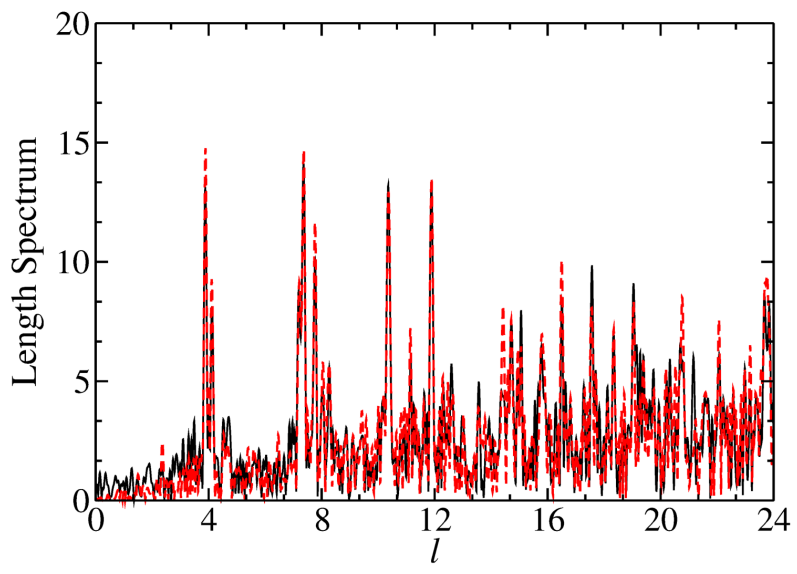

Fig. 6. The same as Fig. 3 for the quarter ellipse QB. with $\Lambda=\sqrt{2 k\left(l_{n, m}^{h}-2 n b\right)}$. The last two sums correspond to the contributions from the stable orbit along the minor axis and the unstable one along the major axis. A detailed derivation of the trace formula can be found in [54]. In Figs. 5 and 6 we compare the fluctuating part of the integrated spectral density and the length spectrum deduced from the eigenvalues of the fully desymmetrized ellipse QB, that is, the quarter ellipse with the Dirichlet boundary conditions with those obtained from the trace formula, that is, the sums over periodic orbits in Eq. (19).

\section{Circular and elliptical NBs}

The Dirac equation of a free, massless spin- $1 / 2$ particle confined to the $(x, y)$ plane reads

$$
\hat{\boldsymbol{H}}_{\mathrm{D}} \boldsymbol{\psi}=E \boldsymbol{\psi}, \boldsymbol{\psi}=\left(\begin{array}{c}
\psi_{1} \\
\psi_{2}
\end{array}\right),
$$

with $\hat{\boldsymbol{H}}_{\mathrm{D}}$ the Dirac Hamiltonian, $\hat{\boldsymbol{H}}_{D}=c \boldsymbol{\sigma} \cdot \boldsymbol{p}$, where $\boldsymbol{p}=\left(p_{x}, p_{y}\right)$ and $\boldsymbol{\sigma}=\left(\hat{\boldsymbol{\sigma}}_{x}, \hat{\boldsymbol{\sigma}}_{y}\right)$ with $\hat{\boldsymbol{\sigma}}_{x, y}$ denoting the Pauli matrices. We are interested in the eigenvalues and eigenfunctions of massless spin- $1 / 2$ particles, which are confined to a bounded two-dimensional convex domain with a smooth boundary which, generally, can be defined in terms of coordinates $0 \leq r \leq \infty$ and $0 \leq \phi \leq 2 \pi$ by a polynomial in the complex plane

$w(z)=x(r, \phi)+\mathrm{i} y(r, \phi)=\sum_{l} c_{l} z^{l}, \quad z=f(r) \mathrm{e}^{\mathrm{i} \phi}$,

with $c_{l}$ real or complex coefficients and some value of $r=r_{0}$ defining the boundary, such that $w(z)$ parametrizes the interior of the billiard domain for $r<r_{0}$. The coordinate transformation from Cartesian coordinates $(x, y)$ to $w(r, \phi)$ yields for the Dirac equation [62]:

$$
k \boldsymbol{\psi}=-\mathrm{i}\left(\begin{array}{c}
0 \\
\frac{1}{z^{\star}\left[w^{\prime}(z)\right]^{*}}\left[\left(\frac{\mathrm{d} f(r)}{\mathrm{d} r}\right)^{-1} f(r) \frac{\partial}{\partial r}+\mathrm{i} \frac{\partial}{\partial \phi}\right] \\
0
\end{array}\right) \psi
$$


Applying the Dirac operator in (22) twice, yields for both wave function components $\psi_{i}, i=1,2$ the Schrödinger Eq. (10):

$$
\begin{gathered}
{\left[\frac{\partial^{2}}{\partial x^{2}}+\frac{\partial^{2}}{\partial y^{2}}\right] \psi_{i}=\left[\left(\frac{\partial}{\partial x}+\mathrm{i} \frac{\partial}{\partial y}\right)\left(\frac{\partial}{\partial x}-\mathrm{i} \frac{\partial}{\partial y}\right)\right] \psi_{i}=} \\
\frac{1}{\left|z w^{\prime}(z)\right|^{2}}\left(\frac{\partial^{2}}{\partial \mu^{2}}+\frac{\partial^{2}}{\partial \phi^{2}}\right) \psi_{i}=k^{2} \psi_{i} .
\end{gathered}
$$

The solutions of Eq. (22) are given by an expansion in terms of plane waves [60]:

$$
\begin{aligned}
& \psi_{1}=\sum_{l} a_{l} \mathrm{i}^{l} J_{l}(k|w(z)|) \mathrm{e}^{\mathrm{i} l \theta(z)}, \\
& \psi_{2}=\sum_{l} b_{l} \mathrm{i}^{l} J_{l}(k|w(z)|) \mathrm{e}^{\mathrm{i} l \theta(z)}
\end{aligned}
$$

with $\mathrm{e}^{\mathrm{i} \theta(z)}=\frac{w(z)}{|w(z)|}$. Inserting this ansatz into the Dirac equation yields

$$
\begin{aligned}
& \sum_{l} a_{l} \mathrm{i}^{l} J_{l}(k|w(z)|) \mathrm{e}^{\mathrm{i} l \theta(z)}= \\
& \sum_{l} b_{l} \mathrm{i}^{l-1} J_{l-1}(k|w(z)|) \mathrm{e}^{\mathrm{i}(l-1) \theta(z)} .
\end{aligned}
$$

Using the orthogonality of these expansions in $\phi$ and $r$ in a circular domain $|w(z)| \leq R_{0}<\infty$ yields the relation [62]:

$$
b_{l}=a_{l-1} \text {. }
$$

Equation (24) provides the solutions of the Dirac equation for a free spin- $1 / 2$ particle in an unbounded system. Those for a massless spin- $1 / 2$ particle confined to a bounded two-dimensional domain of which the boundary is defined at $r=r_{0}$ by $w(\phi)=w\left(r_{0}, \phi\right), \phi \in[0,2 \pi[$ are obtained by imposing the boundary condition that there is no outward current where we chose that for NBs introduced in Ref. [27]. With the notation

$$
\boldsymbol{n}=[\cos \alpha(\phi), \sin \alpha(\phi)]
$$

for the outward normal at the boundary it corresponds to imposing along the boundary the following condition, which yields a relation between the two eigenfunction components:

$$
\psi_{2}(\phi)=\mathrm{ie}^{\mathrm{i} \alpha(\phi)} \psi_{1}(\phi) \equiv \frac{w^{\prime}(\phi)}{\left|w^{\prime}(\phi)\right|} \psi_{1}(\phi) .
$$

For a circular NB we have $f(r)=r$ and thus $w(r, \phi)=r \mathrm{e}^{\mathrm{i} \phi}$, and $\mathrm{e}^{\mathrm{i} \alpha(\phi)}=\mathrm{e}^{\mathrm{i} \phi}$. In this case the eigenvalues and eigenfunctions can be obtained by imposing the boundary condition Eq. (28) directly on the ansatz Eq. (24), yielding the eigenvalues $k_{n}$ of the circular NB as the solutions of the equation [27]:

$$
J_{m+1}\left(k_{n}\right)=J_{m}\left(k_{n}\right)
$$

and for the eigenfunctions

$$
\begin{aligned}
& \psi_{1 m, n}(\boldsymbol{r})=a_{m} \mathrm{i}^{m} J_{m}\left(k_{n} r\right) \mathrm{e}^{\mathrm{i} m \phi}, \\
& \psi_{2 m, n}(\boldsymbol{r})=a_{m} \mathrm{i}^{m+1} J_{m+1}\left(k_{n} r\right) \mathrm{e}^{\mathrm{i}(m+1) \phi} .
\end{aligned}
$$

In Fig. 1 we compare the spectral properties of the circular NB with those of the corresponding QB. In both cases the spectral properties are close to those of Poissonian random numbers.
For a billiard with elliptical shape we choose elliptical coordinates, i.e., $f(\mu)=e^{\mu}$ and $z=e^{\mu} e^{i \phi}$ yielding in the complex-plane notation

$$
\begin{aligned}
& w(z)=\frac{1}{2}\left(\mathrm{e}^{\mu} \mathrm{e}^{\mathrm{i} \phi}+\mathrm{e}^{-\mu} \mathrm{e}^{-\mathrm{i} \phi}\right), \\
& |w(z)|=\sqrt{\cosh ^{2} \mu-\sin ^{2} \phi}, \\
& z w^{\prime}(z)=\frac{1}{2}\left(\mathrm{e}^{\mu} \mathrm{e}^{\mathrm{i} \phi}-\mathrm{e}^{-\mu} \mathrm{e}^{-\mathrm{i} \phi}\right), \\
& \left|z w^{\prime}(z)\right|=\sqrt{\cosh ^{2} \mu-\cos ^{2} \phi} .
\end{aligned}
$$

The boundary of an ellipse with semiminor axis $b=\sinh \mu_{0}$ and semimajor axis $a=\cosh \mu_{0}$ is parametrized by $\phi$ and defined in Eq. (6). The solutions of the Dirac equation are given by Eq. (24) with Eq. (26), yet, they can be expanded in terms of products of the radial and periodic Mathieu functions [57]:

$\psi_{1}(\mu, \phi)=$

$$
\sum_{l} \sum_{n=0}^{\infty}\left\{C e_{n}(\mu) c e_{n}(\phi) \tilde{A}_{n l}+\mathrm{i} S e_{n}(\mu) s e_{n}(\phi) \tilde{B}_{n l}\right\} a_{l},
$$

$\psi_{2}(\mu, \phi)=$

$$
\sum_{l} \sum_{n=0}^{\infty}\left\{C e_{n}(\mu) c e_{n}(\phi) \tilde{A}_{n l}+\mathrm{i} S e_{n}(\mu) s e_{n}(\phi) \tilde{B}_{n l}\right\} a_{l},
$$

$\tilde{A}_{n=2 \nu+\sigma, l=2 \lambda+\sigma}=\mathrm{i}^{-\sigma} \frac{A_{2 \lambda+\sigma}^{2 \nu+\sigma}}{p_{2 \nu+\sigma}}$,

$\tilde{B}_{n=2 \nu+\sigma, l=2 \lambda+\sigma}=\mathrm{i}^{\sigma} \frac{B_{2 \lambda+\sigma}^{2 \nu+\sigma}}{s_{2 \nu+\sigma}}$,

where $\sigma=0$ corresponds to symmteric and $\sigma=1$ to antisymmetric solutions with respect to $\phi \rightarrow \pi+\phi$, where we suppressed the $k$-dependence of $A_{n l}, B_{n l}, p_{n}, s_{n}$; see Eqs. (A16) and (A18). Since there exist several representations of the Mathieu functions we list in Appendix those relevant for the present work. The elliptical billiard exhibits mirror symmetries with respect to the $x$ and $y$ axes. Accordingly, the eigenfunctions of the elliptical quantum billiard may be chosen such that they are either symmetric or antisymmetric with respect to the symmetry axis, that is, follow the Neumann, respectively, the Dirichlet boundary conditions along this axis. This, however, is not the case for NBs [62]. Nevertheless, the shape is mirror symmetric with respect to the minor axis and the major one, which implies a twofold symmetry. Accordingly its eigenfunction components $\psi_{1}$ are either symmetric or antisymmetric with respect to a rotation by $\pi$ implying that, due to the boundary conditions, the components $\psi_{2}$ are antisymmetric or symmetric, corresponding to $\sigma=0$ for $\tilde{A}_{n l}$ and $\sigma=1$ for $\tilde{B}_{n l}$ in Eq. (33), or vice versa. In the following we restrict to the former case.

To obtain the eigenstates of the elliptical NB we have to impose the boundary condition Eq. (28) on the wave function components yielding the quantization condition 


$$
\begin{aligned}
& \sum_{n=0}^{\infty}\left[C e_{2 n+1}\left(\mu_{0}\right) c e_{2 n+1}(\phi) \tilde{a}_{2 n+1}\right. \\
& \left.+\mathrm{i} S e_{2 n+1}\left(\mu_{0}\right) s e_{2 n+1}(\phi) \tilde{b}_{2 n+1}\right]= \\
& \quad \mathrm{i} \mathrm{e}^{\mathrm{i} \alpha(\phi)} \sum_{n=0}^{\infty}\left[C e_{2 n}\left(\mu_{0}\right) c e_{2 n}(\phi) \tilde{a}_{2 n}\right. \\
& \left.\quad+\mathrm{i} S e_{2 n}\left(\mu_{0}\right) s e_{2 n}(\phi) \tilde{b}_{2 n}\right]
\end{aligned}
$$

where we define $\tilde{a}_{2 n+\sigma}=\sum_{l} \tilde{A}_{2 n+\sigma, 2 l+\sigma} a_{2 l}$ and similarly $\tilde{b}_{2 n+\sigma}=\sum_{l} \tilde{B}_{2 n+\sigma, 2 l+\sigma} a_{2 l}$. Furthermore,

$$
\begin{aligned}
& \cos \alpha(\phi)=\frac{\cosh \mu_{0} \cos \phi}{\sqrt{\cosh ^{2} \mu_{0}-\cos ^{2} \phi}}, \\
& \sin \alpha(\phi)=\frac{\sinh \mu_{0} \sin \phi}{\sqrt{\cosh ^{2} \mu_{0}-\cos ^{2} \phi}} .
\end{aligned}
$$

Multiplying the equation with $c e_{2 l+1}(\phi)$, respectively, $s e_{2 l+1}(\phi)$ and integrating over $\phi$ yields with the orthogonality relations (A16) and the notation

$$
\begin{aligned}
& \hat{\boldsymbol{C} \boldsymbol{C}_{l n}}=\int_{-\pi}^{\pi} \mathrm{d} \phi c e_{2 l+1}(\phi) c e_{2 n}(\phi) \cos \alpha(\phi), \\
& \hat{\boldsymbol{S C}_{l n}}=\int_{-\pi}^{\pi} \mathrm{d} \phi s e_{2 l+1}(\phi) c e_{2 n}(\phi) \sin \alpha(\phi), \\
& \hat{\boldsymbol{C S}} \boldsymbol{S}_{l n}=\int_{-\pi}^{\pi} \mathrm{d} \phi c e_{2 l+1}(\phi) s e_{2 n}(\phi) \sin \alpha(\phi), \\
& \hat{\boldsymbol{S} \boldsymbol{S}_{l n}}=\int_{-\pi}^{\pi} \mathrm{d} \phi s e_{2 l+1}(\phi) s e_{2 n}(\phi) \cos \alpha(\phi) \\
& C e_{2 l+1}\left(\mu_{0}\right) \tilde{a}_{2 l+1}=\mathrm{i} \sum_{n=0}^{\infty} \hat{\boldsymbol{C C}}{ }_{l n} C e_{2 n}\left(\mu_{0}\right) \tilde{a}_{2 n} \\
& -\mathrm{i} \sum_{n=0}^{\infty} \hat{\boldsymbol{S C}} \hat{C n}_{l n}\left(\mu_{0}\right) \tilde{b}_{2 n}
\end{aligned}
$$

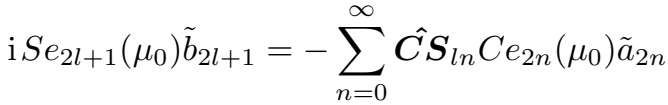

$$
\begin{aligned}
& -\sum_{n=0}^{\infty} \hat{\boldsymbol{S}} \boldsymbol{S}_{l n} S e_{2 n}\left(\mu_{0}\right) \tilde{b}_{2 n}
\end{aligned}
$$

Introducing furthermore the matrices

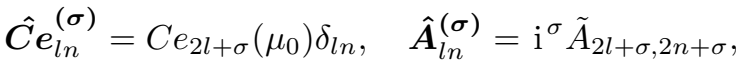

$$
\begin{aligned}
& \hat{\boldsymbol{S} \boldsymbol{e}_{l n}^{(\sigma)}}=S e_{2 l+\sigma}\left(\mu_{0}\right) \delta_{l n}, \quad \hat{\boldsymbol{B}}_{l n}^{(\boldsymbol{\sigma})}=\frac{\tilde{B}_{2 l+\sigma, 2 n+\sigma}}{\mathrm{i}^{\sigma}},
\end{aligned}
$$

and a vector $\boldsymbol{a}$ with components $a_{2 l}$, where $l=1,2, \cdots$, these equations may be brought to the form

$$
\begin{gathered}
\hat{C} e^{(1)} \hat{A}^{(1)} \cdot a=\hat{S C} \cdot \hat{S e^{(0)}} \hat{B}^{(0)} \cdot a \\
-\hat{C C} \cdot \hat{C} e^{(0)} \hat{A}^{(0)} \cdot a,
\end{gathered}
$$

$$
\begin{aligned}
& \hat{S e^{(1)}} \hat{B}^{(1)} \cdot a=\hat{C S} \cdot \hat{C} e^{(0)} \hat{A}^{(0)} \cdot a \\
& \quad+\hat{S S} \cdot \hat{S e^{(0)}} \hat{B}^{(0)} \cdot a,
\end{aligned}
$$

thus yielding the quantization condition

$$
\begin{array}{r}
\hat{S e^{(1)}} \hat{B}^{(1)} \cdot\left(\hat{S C} \cdot \hat{S e^{(0)}} \hat{B}^{(0)}-\hat{C C} \cdot \hat{C} e^{(0)} \hat{A}^{(0)}\right)= \\
\hat{C} e^{(1)} \hat{A}^{(1)} \cdot\left(\hat{C S} \cdot \hat{C} e^{(0)} \hat{A}^{(0)}+\hat{S S} \cdot \hat{S e^{(0)}} \hat{B}^{(0)}\right) .
\end{array}
$$

Equations (39) and (40) have non-trivial solutions if

$$
\begin{aligned}
& \operatorname{det}\left(\hat{C e^{(1)}}-\left(\hat{S C} \cdot \hat{S e^{(0)}} \hat{B}^{(0)}\right.\right. \\
& \left.\left.-\hat{C C} \cdot \hat{C} e^{(0)} \hat{A}^{(0)}\right) \cdot\left(\hat{A}^{(1)}\right)^{-1}\right)=0 \\
& \operatorname{det}\left(\hat{S e^{(1)}}-\left(\hat{C S} \cdot \hat{C} e^{(0)} \hat{A}^{(0)}\right.\right. \\
& \left.\left.+\hat{S S} \cdot \hat{S e}^{(0)} \hat{B}^{(0)}\right) \cdot\left(\hat{B}^{(1)}\right)^{-1}\right)=0
\end{aligned}
$$

Thus the eigenvalues are obtained from a relation between the Mathieu functions $C e_{2 l+1}\left(\mu_{0}\right), S e_{2 l+1}\left(\mu_{0}\right)$ and linear combinations of $C e_{2 n}\left(\mu_{0}\right), S e_{2 n}\left(\mu_{0}\right)$. In Fig. 4 we compare the spectral properties for the elliptical NB with those for the corresponding QB. Both exhibit a behavior close to Poissonian statistics. Furthermore, we show a few examples of the wave function components $\psi_{1}$ and $\psi_{2}$ in Figs. 7 and 8.

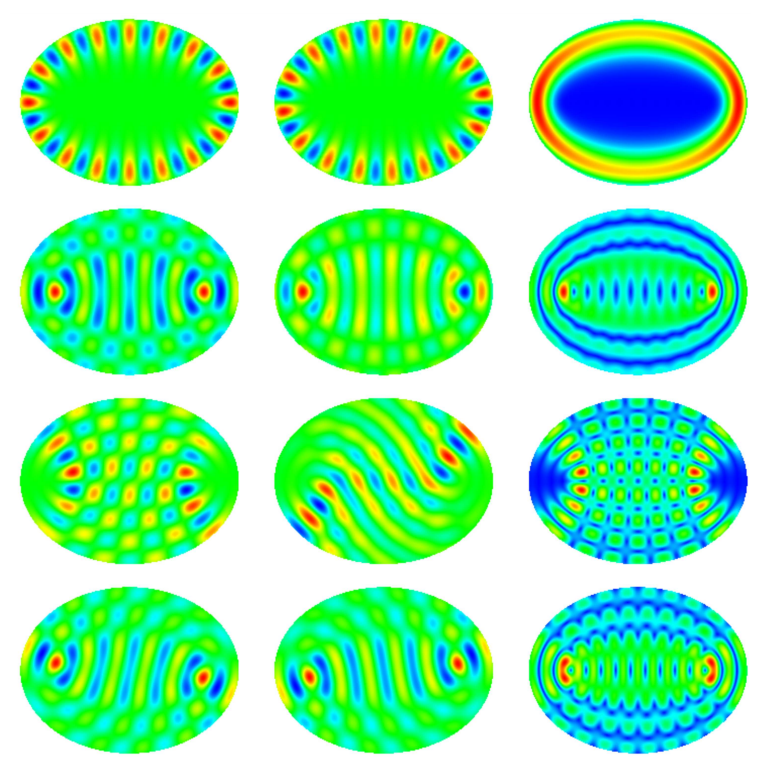

Fig. 7. The real part (first column), imaginary part (second column) and modulus (third column) of the wave function component $\Psi_{1}$ of the eigenstates with $n=101,102,105,106$ from up to down. The color code ranges from dark blue for the minimum value, to dark red for the maximal one. 


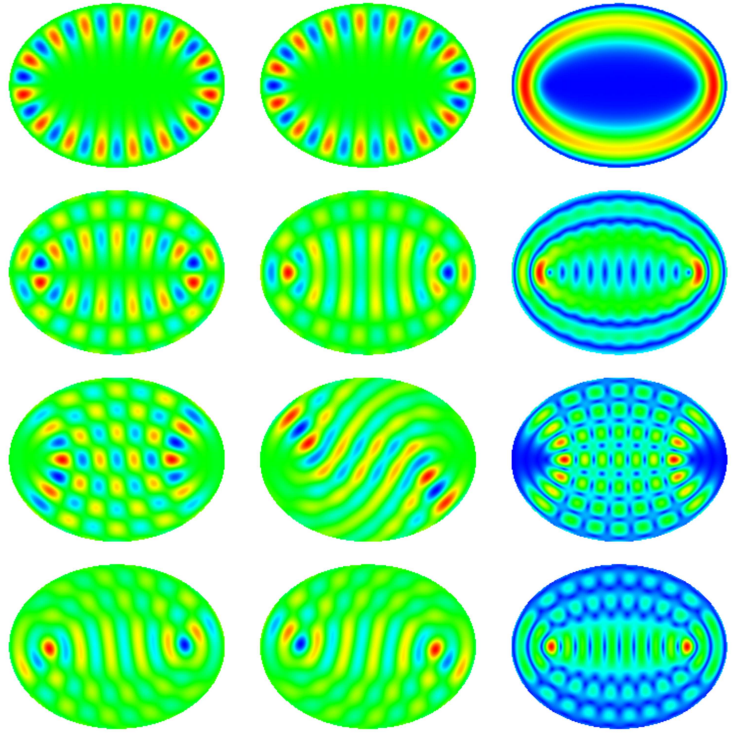

Fig. 8. The real part (first column), imaginary part (second column) and modulus (third column) of the wave function component $\Psi_{2}$ of the eigenstates with $n=101,102,105,106$ from up to down. The color code ranges from dark blue for the minimum value to dark red for the maximal one.

Note that $\hat{\boldsymbol{H}}_{D}(-x, y)=\hat{\boldsymbol{H}}_{D}^{\star}(x, y)$ and $\hat{\boldsymbol{H}}_{D}(x,-y)=$ $\hat{\boldsymbol{\sigma}}_{\boldsymbol{z}} \hat{\boldsymbol{H}}_{D}^{\star}(x, y) \hat{\boldsymbol{\sigma}}_{\boldsymbol{z}}$, and the corresponding eigenfunctions fulfill the boundary condition (28) if those of $\hat{\boldsymbol{H}}_{D}(x, y)$ do. Thus the set of eigenfunctions of the elliptical billiards will comprise some with the property $\left[\psi_{1}(-x, y), \psi_{2}(-x, y)\right]= \pm\left[\psi_{1}^{\star}(x, y), \psi_{2}^{\star}(x, y)\right]$, and $\left[\psi_{1}(x,-y), \psi_{2}(x,-y)\right]=\left[ \pm \psi_{1}^{\star}(x, y), \mp \psi_{2}^{\star}(x, y)\right]$, respectively, which corresponds to real coefficients $a_{l}$ in Eq. (33). In the first two rows of Figs. 7 and 8 examples of the wave function components are shown, which exhibit this relation.

\section{Derivation of a trace formula from the boundary integral equation for the eigenvalues of NBs}

In order to derive semiclassical approximations for the spectral densities of circular and elliptical NBs, which express them in terms of trace formulae, i.e., a sum over periodic orbits of the corresponding CBs, we proceed as in [47] and therefore first briefly review that derivation. The starting point was a boundary integral equation originating from the Green theorem, which provides an exact integral equation for the eigenvalues and the associated eigenfunctions in terms of the wave functions along the boundary. It has the advantage that the eigenvalue problem is reduced from a two-dimensional integral over the billiard area to a one-dimensional one along the boundary. For the interior Dirichlet problem the eigenvalues are obtained from a Fredholm equation of the second kind which involves the normal derivative $u(s)=\partial_{n} \psi(s)$ instead of the wave functions $\psi(s)$ themselves with the arc length $s$ parametrizing the boundary [64]:

$$
\begin{aligned}
& u\left(s^{\prime}\right)=-2 \oint_{\partial \Omega} \mathrm{d} s \partial_{n^{\prime}} G_{0}\left[\boldsymbol{r}\left(s^{\prime}\right), \boldsymbol{r}(s) ; k\right] u(s)= \\
& \hat{Q}(k) u(s),
\end{aligned}
$$

where

$$
\begin{aligned}
& \left\langle\boldsymbol{r}^{\prime}\left|\left[(k+\mathrm{i} \epsilon)^{2}+\Delta\right]^{-1}\right| \boldsymbol{r}\right\rangle \equiv G_{0}\left[\boldsymbol{r}\left(s^{\prime}\right), \boldsymbol{r}(s)\right]= \\
& -\mathrm{i} / 4 H_{0}^{(1)}\left(k\left|\boldsymbol{r}-\boldsymbol{r}^{\prime}\right|\right)
\end{aligned}
$$

denotes the free-space Green function in two-dimensions and $H_{0}^{(1)}(x)$ the Hankel function of order zero of the first kind. Its derivative with respect to the normal vector $\boldsymbol{n}(s)=[\cos \alpha(s), \sin \alpha(s)]$ equals

$\partial_{n^{\prime}} G_{0}\left[\boldsymbol{r}\left(s^{\prime}\right), \boldsymbol{r}(s) ; k\right]=$

$$
\begin{aligned}
& \hat{\boldsymbol{n}}\left(s^{\prime}\right) \cdot \frac{\boldsymbol{r}(s)-\boldsymbol{r}\left(s^{\prime}\right)}{\left|\boldsymbol{r}(s)-\boldsymbol{r}\left(s^{\prime}\right)\right|}\left(-\frac{\mathrm{i}}{4} k\right) H_{1}^{(1)}\left(k\left|\boldsymbol{r}(s)-\boldsymbol{r}\left(s^{\prime}\right)\right|\right)= \\
& \cos \left(\alpha\left(s^{\prime}\right)-\xi\left(s, s^{\prime}\right)\right)\left(-\frac{\mathrm{i}}{4} k\right) H_{1}^{(1)}(k \rho),
\end{aligned}
$$

with the notations $\rho=\left|\boldsymbol{r}(s)-\boldsymbol{r}\left(s^{\prime}\right)\right|$ and $\xi\left(s, s^{\prime}\right)$ denoting the angle between the $x$-axis and $\boldsymbol{\rho}\left(s, s^{\prime}\right)=\boldsymbol{r}(s)-\boldsymbol{r}\left(s^{\prime}\right)$. Equation (44) has non-trivial solutions only if

$$
\operatorname{det}(\mathbb{1}-\hat{Q}(k))=0 \text {. }
$$

The determinant has zeroes for discrete values of $k$ which yield the eigenwavenumbers $k_{n}$ and thus the eigenvalues $E_{n}=k_{n}^{2}$ of the quantum billiard. Accordingly, the integrated spectral density, that is, the number of eigenvalues below a given value $E=k^{2}$ can be obtained from

$$
\begin{aligned}
& N(E)=N^{\text {smooth }}(E) \\
& -\frac{1}{\pi} \lim _{\epsilon \rightarrow 0+} \operatorname{Im} \ln \frac{\operatorname{det}(\mathbb{1}-\hat{Q}(k))(E+i \epsilon)}{\operatorname{det}(\mathbb{1}-\hat{Q}(k))(0)} .
\end{aligned}
$$

The spectral density $\rho(k)$ is given by the derivative of $N(k)$ with respect to $k, \rho(k)=\frac{\mathrm{d}}{\mathrm{d} k} N(k)$, yielding for the fluctuating part

$$
\begin{gathered}
\rho^{\mathrm{fluc}}(k)=-\frac{1}{\pi} \operatorname{Im} \frac{\mathrm{d}}{\mathrm{d} k} \ln \operatorname{det}(\mathbb{1}-\hat{Q}(k))= \\
\frac{1}{\pi} \operatorname{Im} \sum_{p=1}^{\infty} \frac{1}{p} \frac{\mathrm{d}}{\mathrm{d} k}\left(\operatorname{Tr} \hat{Q}^{p}(k)\right),
\end{gathered}
$$

where we used the property $\operatorname{det} \hat{M}=\exp (\operatorname{Tr} \ln \hat{M})$ and

$$
\begin{aligned}
& \operatorname{Tr} \hat{Q}^{p}(k)=(-2)^{p} \oint_{\partial \Omega} \mathrm{d} s_{1} \oint_{\partial \Omega} \mathrm{d} s_{2} \cdots \oint_{\partial \Omega} \mathrm{d} s_{p} \\
& \quad \times \partial_{n_{1}} G_{0}\left[\boldsymbol{r}\left(s_{1}\right), \boldsymbol{r}\left(s_{2}\right) ; k\right] \partial_{n_{2}} G_{0}\left[\boldsymbol{r}\left(s_{2}\right), \boldsymbol{r}\left(s_{3}\right) ; k\right] \cdots \\
& \quad \times \partial_{n_{p}} G_{0}\left[\boldsymbol{r}\left(s_{p}\right), \boldsymbol{r}\left(s_{1}\right) ; k\right] .
\end{aligned}
$$


Note that $N^{\text {smooth }}(E)$ might contain besides the usual Weyl term slowly oscillating contributions. The integrals were evaluated in stationary phase approximation in Ref. [47] yielding Gutzwiller's trace formula for quantum billiards of which the corresponding classical dynamics is chaotic.

The boundary integral equation was generalized to NBs in Ref. [27] based on the Dirac equation for $\boldsymbol{\psi}^{\dagger}$ :

$$
k \boldsymbol{\psi}^{\dagger}(\boldsymbol{r})-\mathrm{i} \boldsymbol{\nabla}_{\boldsymbol{r}} \boldsymbol{\psi}^{\dagger}(\boldsymbol{r}) \cdot \hat{\boldsymbol{\sigma}}=0
$$

for $\boldsymbol{\psi}=\left(\begin{array}{c}\psi_{1} \\ \psi_{2}\end{array}\right)$ and the matrix equation for the associated free-space Green operator, which acts on spinors and thus is a $2 \times 2$ matrix in coordinate space,

$$
k \hat{\boldsymbol{G}}_{\mathbf{0}}\left(\boldsymbol{r}, \boldsymbol{r}^{\prime}\right)+\mathrm{i} \hat{\boldsymbol{\sigma}} \cdot \boldsymbol{\nabla}_{\boldsymbol{r}} \hat{\boldsymbol{G}}_{\mathbf{0}}\left(\boldsymbol{r}, \boldsymbol{r}^{\prime}\right)=\delta\left(\boldsymbol{r}-\boldsymbol{r}^{\prime}\right) \mathbb{1},
$$

where $\mathbb{1}$ is a $2 \times 2$ unit matrix and the Green function $\hat{G}_{\mathbf{0}}\left(\boldsymbol{r}, \boldsymbol{r}^{\prime}\right)$ refers to the coordinate representation of the Green operator $\hat{\boldsymbol{G}}=(k+\mathrm{i} \epsilon+\mathrm{i} \hat{\boldsymbol{\sigma}} \cdot \boldsymbol{\nabla})^{-1}$, $\hat{\boldsymbol{G}}_{\mathbf{0}}\left(\boldsymbol{r}, \boldsymbol{r}^{\prime}\right)=\left\langle\boldsymbol{r}|\hat{\boldsymbol{G}}| \boldsymbol{r}^{\prime}\right\rangle$. In terms of the coordinate system introduced in Eq. (21) this yields [62] with the notations $z=f(r) \mathrm{e}^{\mathrm{i} \phi}, z^{\prime}=f\left(r^{\prime}\right) \mathrm{e}^{\mathrm{i} \phi^{\prime}}, \boldsymbol{\rho}\left(z, z^{\prime}\right)=w(z)-w\left(z^{\prime}\right)$, $\rho\left(z, z^{\prime}\right)=\left|\boldsymbol{\rho}\left(z, z^{\prime}\right)\right|$, and $\mathrm{e}^{\mathrm{i} \xi\left(z, z^{\prime}\right)}=\frac{\boldsymbol{\rho}\left(z, z^{\prime}\right)}{\rho\left(z, z^{\prime}\right)}$ for the freespace Green function

$$
\begin{aligned}
& \hat{\boldsymbol{G}}_{\mathbf{0}}\left(\boldsymbol{r}, \boldsymbol{r}^{\prime}\right)= \\
& \quad \frac{k}{4}\left(\begin{array}{cc}
-\mathrm{i} H_{0}^{(1)}\left[k \rho\left(z, z^{\prime}\right)\right] & \mathrm{e}^{-\mathrm{i} \xi\left(z, z^{\prime}\right)} H_{1}^{(1)}\left[k \rho\left(z, z^{\prime}\right)\right] \\
\mathrm{e}^{\mathrm{i} \xi\left(z, z^{\prime}\right)} H_{1}^{(1)}\left[k \rho\left(z, z^{\prime}\right)\right] & -\mathrm{i} H_{0}^{(1)}\left[k \rho\left(z, z^{\prime}\right)\right]
\end{array}\right) .
\end{aligned}
$$

The spectral density $\rho(k)$ is given in terms of the trace of the outgoing Green operator of the NB which complies with the boundary conditions and reads in coordinate space

$$
\begin{aligned}
& \left\langle\boldsymbol{r}|\hat{\boldsymbol{G}}| \boldsymbol{r}^{\prime}\right\rangle=\left(\begin{array}{cc}
G_{11}\left(\boldsymbol{r}, \boldsymbol{r}^{\prime} ; k\right) & G_{12}\left(\boldsymbol{r}, \boldsymbol{r}^{\prime} ; k\right) \\
G_{21}\left(\boldsymbol{r}, \boldsymbol{r}^{\prime} ; k\right) & G_{22}\left(\boldsymbol{r}, \boldsymbol{r}^{\prime} ; k\right)
\end{array}\right), \\
& \rho(k)=-\frac{1}{\pi} \iint_{\Omega} \mathrm{d} \boldsymbol{r} \operatorname{Im}\left(G_{11}(\boldsymbol{r}, \boldsymbol{r})+G_{22}(\boldsymbol{r}, \boldsymbol{r})\right) .
\end{aligned}
$$

The boundary integral equation for NBs corresponding to that for QBs Eq. (44) reads

$\boldsymbol{\psi}^{\dagger}\left[\boldsymbol{r}\left(s^{\prime}\right)\right]=2 \mathrm{i} \oint_{\partial \Omega} \mathrm{d} s \hat{\boldsymbol{n}} \cdot\left\{\left[\boldsymbol{\psi}(\boldsymbol{r}(s)]^{\dagger} \hat{\boldsymbol{\sigma}} \hat{\boldsymbol{G}}_{\mathbf{0}}\left[\boldsymbol{r}(s), \boldsymbol{r}\left(s^{\prime}\right) ; k\right]\right\}=\right.$

$$
\hat{\boldsymbol{Q}}(k) \boldsymbol{\psi}^{\dagger}[\boldsymbol{r}(s)] \text {. }
$$

This yields two coupled equations for the wave function components $\psi_{1}(s), \psi_{2}(s)$, which have non-trivial solutions if

$$
\operatorname{det}(\mathbb{1}-\hat{\boldsymbol{Q}}(k))=0 .
$$

The integrand contains singularities which are due to those of the two Hankel functions at $\rho=0$. Two independent equations are obtained for them by applying the boundary condition Eq. (28) to these equations. The singularities may be removed by adding, respectively, subtracting the resulting equations from each other yielding a set of equations for the eigenwavenumbers $k_{n}$ :

$$
\psi_{1}^{\star}\left(s^{\prime}\right)=\oint_{\partial \Omega} \mathrm{d} s Q^{(1)}\left(s^{\prime}, s ; k\right) \psi_{1}^{\star}(s)
$$

with

$$
\begin{aligned}
& Q^{(1)}\left(s^{\prime}, s ; k\right)=\left[\mathrm{e}^{\mathrm{i}\left[\alpha\left(s^{\prime}\right)-\alpha(s)\right]}-1\right] \frac{\mathrm{i} k}{4} H_{0}^{(1)}\left[k \rho\left(s, s^{\prime}\right)\right] \\
& +\left[\mathrm{e}^{\mathrm{i} \alpha\left(s^{\prime}\right)-\mathrm{i} \xi\left(s, s^{\prime}\right)}+\mathrm{e}^{-\mathrm{i} \alpha(s)+\mathrm{i} \xi\left(s, s^{\prime}\right)}\right] \frac{\mathrm{i} k}{4} H_{1}^{(1)}\left[k \rho\left(s, s^{\prime}\right)\right]
\end{aligned}
$$

and

$$
\psi_{2}^{\star}\left(s^{\prime}\right)=\oint_{\partial \Omega} \mathrm{d} s Q^{(2)}\left(s^{\prime}, s ; k\right) \psi_{2}^{\star}(s)
$$

with

$$
\begin{aligned}
& Q^{(2)}\left(s^{\prime}, s ; k\right)=\left[1-\mathrm{e}^{\mathrm{i}\left[\alpha(s)-\alpha\left(s^{\prime}\right)\right]}\right] \frac{\mathrm{i} k}{4} H_{0}^{(1)}\left[k \rho\left(s, s^{\prime}\right)\right] \\
& +\left[\mathrm{e}^{\mathrm{i} \alpha(s)-\mathrm{i} \xi\left(s, s^{\prime}\right)}+\mathrm{e}^{-\mathrm{i} \alpha\left(s^{\prime}\right)+\mathrm{i} \xi\left(s, s^{\prime}\right)}\right] \frac{\mathrm{i} k}{4} H_{1}^{(1)}\left[k \rho\left(s, s^{\prime}\right)\right] .
\end{aligned}
$$

Note that both integrands approach zero when $s \rightarrow s^{\prime}$. Furthermore, both equations yield the same set of solutions for the eigenwavenumbers $k_{n}$. Proceeding as in the non-relativistic case we may again express the fluctuating part of the spectral density as in Eq. (49) in terms of a trace of $\hat{\boldsymbol{Q}}^{p}(k)$, where

$$
\begin{aligned}
\operatorname{Tr} & \hat{\boldsymbol{Q}}^{p}(k)=\oint_{\partial \Omega} \mathrm{d} s_{1} \oint_{\partial \Omega} \mathrm{d} s_{2} \cdots \oint_{\partial \Omega} \mathrm{d} s_{p} \\
& \times Q^{(1)}\left[\boldsymbol{r}\left(s_{1}\right), \boldsymbol{r}\left(s_{2}\right) ; k\right] Q^{(1)}\left[\boldsymbol{r}\left(s_{2}\right), \boldsymbol{r}\left(s_{3}\right) ; k\right] \cdots \\
& \times Q^{(1)}\left[\boldsymbol{r}\left(s_{p}\right), \boldsymbol{r}\left(s_{1}\right) ; k\right] \\
& +\oint_{\partial \Omega} \mathrm{d} s_{1} \oint_{\partial \Omega} \mathrm{d} s_{2} \cdots \oint_{\partial \Omega} \mathrm{d} s_{p} \\
& \times Q^{(2)}\left[\boldsymbol{r}\left(s_{1}\right), \boldsymbol{r}\left(s_{2}\right) ; k\right] Q^{(2)}\left[\boldsymbol{r}\left(s_{2}\right), \boldsymbol{r}\left(s_{3}\right) ; k\right] \cdots \\
& \times Q^{(2)}\left[\boldsymbol{r}\left(s_{p}\right), \boldsymbol{r}\left(s_{1}\right) ; k\right] .
\end{aligned}
$$

We are interested in the semiclassical limit, i.e. in $k \rightarrow \infty$, where

$$
G_{0}\left[\boldsymbol{r}(s), \boldsymbol{r}\left(s^{\prime}\right) ; k\right]=-\frac{\mathrm{i}}{4} H_{0}^{(1)}(k \rho) \simeq-\frac{\mathrm{i}}{4} \sqrt{\frac{2}{\pi \rho}} \mathrm{e}^{\mathrm{i} k \rho-\frac{\mathrm{i}}{4} \pi}
$$

and

$$
H_{1}(k \rho) \simeq \frac{1}{\mathrm{i}} H_{0}(k \rho),
$$

so that

$$
\begin{aligned}
& \partial_{n^{\prime}} G_{0}\left[\boldsymbol{r}(s), \boldsymbol{r}\left(s^{\prime}\right) ; k\right] \simeq \\
& \quad \mathrm{i} \cos \left(\alpha\left(s^{\prime}\right)-\xi\left(s, s^{\prime}\right)\right)(-k) G_{0}\left[\boldsymbol{r}(s), \boldsymbol{r}\left(s^{\prime}\right) ; k\right] .
\end{aligned}
$$

Replacing in Eq. (62) the Hankel functions by these large- $k$ approximations yields 


$$
\begin{aligned}
& \operatorname{Tr} \hat{\boldsymbol{Q}}^{p}(k)=\left(\frac{1}{i}\right)^{p} \oint_{\partial \Omega} \mathrm{d} s_{1} \oint_{\partial \Omega} \mathrm{d} s_{2} \cdots \oint_{\partial \Omega} \mathrm{d} s_{p} \\
& \quad \times\left\{\prod_{j=1}^{p} \frac{\left[\mathrm{e}^{\mathrm{i}\left[\alpha\left(s_{j}\right)-\alpha\left(s_{j+1}\right)\right]}-1\right]-\mathrm{i}\left[\mathrm{e}^{\mathrm{i} \alpha\left(s_{j}\right)-\mathrm{i} \xi\left(s_{j}, s_{j+1}\right)}+\mathrm{e}^{-\mathrm{i} \alpha\left(s_{j+1}\right)+\mathrm{i} \xi\left(s_{j}, s_{j+1}\right)}\right]}{\cos \left(\alpha\left(s_{j}\right)-\xi\left(s_{j+1}, s_{j}\right)\right)}\right. \\
& \left.+\prod_{j=1}^{p} \frac{\left[1-\mathrm{e}^{\mathrm{i}\left[\alpha\left(s_{j+1}\right)-\alpha\left(s_{j}\right)\right]}\right]-\mathrm{i}\left[\mathrm{e}^{\mathrm{i} \alpha\left(s_{j+1}\right)-\mathrm{i} \xi\left(s_{j}, s_{j+1}\right)}+\mathrm{e}^{-\mathrm{i} \alpha\left(s_{j}\right)+\mathrm{i} \xi\left(s_{j}, s_{j+1}\right)}\right]}{\cos \left(\alpha\left(s_{j}\right)-\xi\left(s_{j+1}, s_{j}\right)\right)}\right\} \\
& \quad \times \partial_{n_{1}} G_{0}\left[\boldsymbol{r}\left(s_{1}\right), \boldsymbol{r}\left(s_{2}\right) ; k\right] \partial_{n_{2}} G_{0}\left[\boldsymbol{r}\left(s_{2}\right), \boldsymbol{r}\left(s_{3}\right) ; k\right] \cdots \partial_{n_{p}} G_{0}\left[\boldsymbol{r}\left(s_{p}\right), \boldsymbol{r}\left(s_{1}\right) ; k\right],
\end{aligned}
$$

where $s_{p+1}=s_{1}$ and $\partial_{n_{i}}=\boldsymbol{n}_{i} \cdot \boldsymbol{\nabla}$ with $\boldsymbol{n}_{i}$ denoting the normal to the boundary at $s=s_{i}$. In Ref. [47] the integrals were performed by replacing the Green functions by their approximate values for large $k$ given in Eqs. (63) and (65) and using the stationary phase approximation. However, due to the existence of continuous families of periodic orbits $[53,54]$, the latter leads to diverging integrals for the circular and the elliptical billiards. Yet, we may proceed as in [53], that is, assume that the dominant contributions to $\operatorname{Tr} \hat{Q}^{p}(k)$ in Eq. (66) come from the periodic orbits, whereas those of nonperiodic closed orbits may be neglected. This would correspond to accounting in the products in Eq. (66) only for periodic orbits of length $p$, so that they do not depend on the integration variables but on the choice of the periodic orbits of length $p$. This corresponds to anticipating the results of the stationary phase approximation leading to

$$
\begin{aligned}
& \operatorname{Tr} \hat{\boldsymbol{Q}}^{p}(k) \simeq\left\{\left(\frac{1}{\mathrm{i}}\right)^{p} \prod_{j=1}^{p} \frac{\left[\mathrm{e}^{\mathrm{i}\left[\alpha_{j}-\alpha_{j+1}\right]}-1\right]-\mathrm{i}\left[\mathrm{e}^{\mathrm{i} \alpha_{j}-\mathrm{i} \xi_{j+1, j}}+\mathrm{e}^{-\mathrm{i} \alpha_{j+1}+\mathrm{i} \xi_{j, j+1}}\right]}{\cos \left(\xi_{j, j-1}-\alpha_{j}\right)}\right. \\
& \left.+\left(\frac{1}{\mathrm{i}}\right)^{p} \prod_{j=1}^{p} \frac{\left[1-\mathrm{e}^{\mathrm{i}\left[\alpha_{j+1}-\alpha_{j}\right]}\right]-\mathrm{i}\left[\mathrm{e}^{\mathrm{i} \alpha_{j+1}-\mathrm{i} \xi_{j, s_{j+1}}}+\mathrm{e}^{-\mathrm{i} \alpha_{j}+\mathrm{i} \xi_{j+1, j}}\right]}{\cos \left(\xi_{j, j-1}-\alpha_{j}\right)}\right\} \oint_{\partial \Omega} \mathrm{d} s_{1} \oint_{\partial \Omega} \mathrm{d} s_{2} \cdots \oint_{\partial \Omega} \mathrm{d} s_{p} \\
& \quad \times \partial_{n_{1}} G_{0}\left[\boldsymbol{r}\left(s_{1}\right), \boldsymbol{r}\left(s_{2}\right) ; k\right] \partial_{n_{2}} G_{0}\left[\boldsymbol{r}\left(s_{2}\right), \boldsymbol{r}\left(s_{3}\right) ; k\right] \cdots \partial_{n_{p}} G_{0}\left[\boldsymbol{r}\left(s_{p}\right), \boldsymbol{r}\left(s_{1}\right) ; k\right],
\end{aligned}
$$

where the products run over the points $j=1, \cdots, p$ of impact with the boundary of a periodic orbit with period $p$. Here, we used that

$$
\xi_{j, j-1}+\xi_{j+1, j}=2 \alpha_{j}+\pi,
$$

where $\xi_{j, j-1}-\alpha_{j}$ is the angle between the incoming trajectory and the normal at the $j$-th point of impact. The remaining integrals were evaluated exactly in Ref. [53] for the circular quantum billiards.

The two products in the prefactor can be further simplified. The factors in the first product can be brought to the form

$$
\begin{gathered}
{\left[\mathrm{e}^{\mathrm{i} \alpha_{j}-\mathrm{i} \alpha_{j+1}}-1\right]-\mathrm{i}\left[\mathrm{e}^{\mathrm{i} \alpha_{j}-\mathrm{i} \xi_{j+1, j}}+\mathrm{e}^{-\mathrm{i} \alpha_{j+1}+\mathrm{i} \xi_{j+1, j}}\right]=} \\
\left(\mathrm{e}^{\mathrm{i} \alpha_{j}-\mathrm{i} \xi_{j+1, j}}-\mathrm{i}\right) \cdot\left(\mathrm{e}^{-\mathrm{i} \alpha_{j+1}+\mathrm{i} \xi_{j+1, j}}-\mathrm{i}\right) \cdot
\end{gathered}
$$

Using the equality Eq. (67) the first factor can be expressed as $-\left(\mathrm{e}^{\mathrm{i} \xi_{j, j-1}-\mathrm{i} \alpha_{j}}+\mathrm{i}\right)$, where $\xi_{0,1}=\xi_{p, 1}$.
Then the product becomes

$$
\begin{aligned}
& \mathrm{i}^{p} \prod_{j=1}^{p}\left(\mathrm{e}^{\mathrm{i} \xi_{j, j-1}-\mathrm{i} \alpha_{j}}+\mathrm{i}\right) \cdot\left(\mathrm{e}^{-\mathrm{i} \alpha_{j+1}+\mathrm{i} \xi_{j+1, j}}-\mathrm{i}\right)= \\
& \quad(-2)^{p} \prod_{j=1}^{p} \mathrm{e}^{\mathrm{i} \xi_{j, j-1}-\mathrm{i} \alpha_{j}-\mathrm{i} \pi / 2} \cos \left[\xi_{j, j-1}-\alpha_{j}\right] .
\end{aligned}
$$

The factors in the second product coincide with those of the first one up to a phase factor $\mathrm{e}^{\mathrm{i} \alpha_{j+1}-\mathrm{i} \alpha_{j}}=$ $\mathrm{e}^{\mathrm{i}\left[\alpha_{j+1}-\xi_{j+1, j}+\alpha_{j}-\xi_{j, j-1}+\pi\right]}$ so that Eq. (66) becomes

$$
\begin{aligned}
\operatorname{Tr} \hat{\boldsymbol{Q}}^{p}(k) \simeq 2 \cos \Phi_{p} \oint_{\partial \Omega} \mathrm{d} s_{1} \oint_{\partial \Omega} \mathrm{d} s_{2} \cdots \oint_{\partial \Omega} \mathrm{d} s_{p} \\
\quad \times(-2)^{p} \partial_{n_{1}} G_{0}\left[\boldsymbol{r}\left(s_{1}\right), \boldsymbol{r}\left(s_{2}\right) ; k\right] \partial_{n_{2}} G_{0}\left[\boldsymbol{r}\left(s_{2}\right), \boldsymbol{r}\left(s_{3}\right) ; k\right] \cdots \\
\quad \times \partial_{n_{p}} G_{0}\left[\boldsymbol{r}\left(s_{p}\right), \boldsymbol{r}\left(s_{1}\right) ; k\right]
\end{aligned}
$$


where

$$
\begin{gathered}
\Phi_{p}=\sum_{j=1}^{p}\left[\xi_{j, j-1}-\alpha_{j}-\pi / 2\right] \equiv \\
\sum_{j=1}^{p} \frac{\left[\xi_{j, j-1}-\xi_{j+1, j}\right]}{2}=\frac{\xi_{1,0}-\xi_{p+1, p}}{2} .
\end{gathered}
$$

The phase $\Phi_{p}$ can be further evaluated by recursively applying Eq. (67), leading to [27]:

$$
\begin{aligned}
& \xi_{p+1, p}-\xi_{1,0}=\pi+2 \alpha_{p}-\xi_{p, p-1}-\xi_{1,0}= \\
& \quad \sum_{n=1}^{p}(-1)^{p-n}\left[\pi+2 \alpha_{n}\right]+(-1)^{p} \xi_{1,0}-\xi_{1,0}= \\
& \quad \begin{cases}2 \sum_{n=1}^{p / 2}\left[\alpha_{2 n}-\alpha_{2 n-1}\right], & p \text { even }, \\
2 \sum_{n=1}^{(p+1) / 2}\left[\alpha_{2 n-1}-\alpha_{2 n}\right]+\pi-2 \xi_{1,0}, & p \text { odd } .\end{cases}
\end{aligned}
$$

The sum in Eq. (49) is over clockwise and counterclockwise propagating periodic orbits. For a QB both contributions coincide, whereas NBs exhibit a chirality property, that is $\Phi_{p}$ depends on the rotational direction. Reversing it corresponds in Eq. (72) to exchange of the even and odd indices of the angle $\alpha$ for $p$ even, whereas for $p$ odd just the value of the initial angle $\xi_{0,1}$ changes to $\xi_{0,1}+\pi$ [27], or, equivalently, in Eq. (71) to replacing $\xi_{j, j-1}-\alpha_{j}$ by $-\left(\xi_{j, j-1}-\alpha_{j}\right)$. Accordingly, the phase for the reversed rotational direction, $\tilde{\Phi}_{p}$, becomes

$$
\tilde{\Phi}_{p}=\sum_{j=1}^{p}\left[-\left(\xi_{j, j-1}-\alpha_{j}\right)-\pi / 2\right] .
$$

Consequently, summing over clockwise and counterclockwise contributions of Eq. (70) yields for the prefactor

$$
\cos \Phi_{p}+\cos \tilde{\Phi}_{p}=2 \cos \left(\sum_{j=1}^{p}\left[\xi_{j, j-1}-\alpha_{j}\right]\right) \cos \left(p \frac{\pi}{2}\right) .
$$

Hence, contributions with odd $p$ cancel each other and the sum over the periodic orbits can be restricted to those with an even number of reflections. Since the sum in Eq. (70) is over periodic orbits, the total phase accumulated by the trajectory with points of reflection at $\xi_{j+1, j}, j=1, \cdots, 2 p$, which returns to the initial point of reflection after $2 p+1$ reflections, corresponds to a multiple of $2 \pi, \xi_{2 p+1,2 p}-\xi_{1,0}=m_{\varphi} 2 \pi$, where $m_{\varphi}$ denotes the number of rotations performed during the evolution. Accordingly, $\Phi_{p}=m_{\varphi} \pi$. Denoting by $\rho^{\text {osc }}(k)=\sum_{p=1}^{\infty} \sum_{\gamma_{p}} \mathcal{A}_{\gamma_{p}} \mathrm{e}^{\mathrm{i} \beta_{\gamma_{p}}}$, where the second sum runs over all periodic orbits $\gamma_{p}$ with number of reflections $p$, the trace formula of the corresponding $\mathrm{QB}$, we finally obtain for that of the NB

$$
\rho^{\mathrm{osc}}(k)=\sum_{p=1}^{\infty} \sum_{\gamma_{2 p}}(-1)^{p} \cos \left(m_{\varphi_{2 p}} \pi\right) \mathcal{A}_{\gamma_{2 p}} \mathrm{e}^{\mathrm{i} \beta_{\gamma_{2 p}}} .
$$

The trace formula for NBs thus differs from that of the corresponding QB through the additional phase factor and the fact that only periodic orbits with an even number of reflections at the boundary contribute [49].

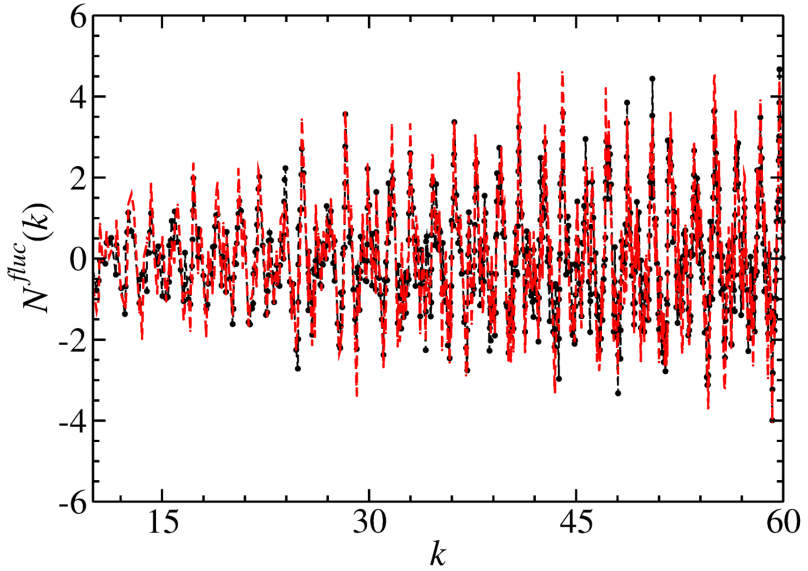

Fig. 9. The same as Fig. 2 for the circular NB.

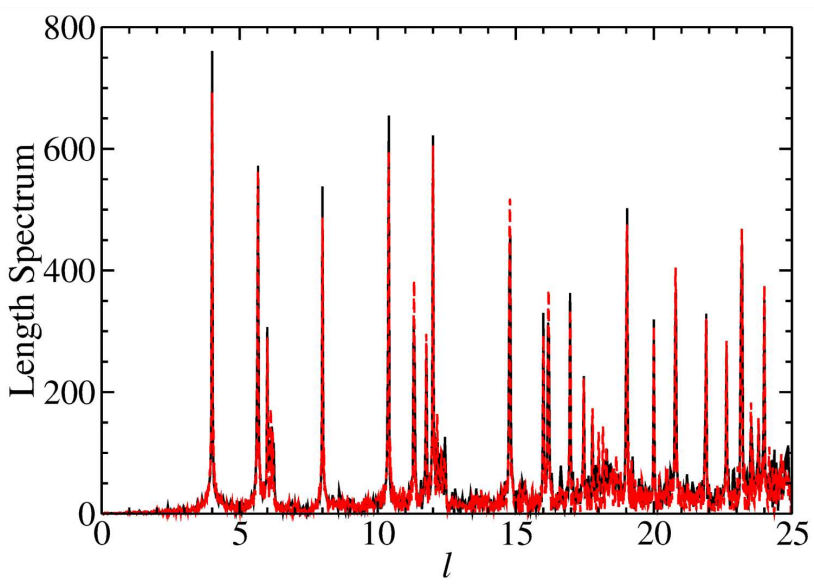

Fig. 10. The same as Fig. 3 for the circular NB.

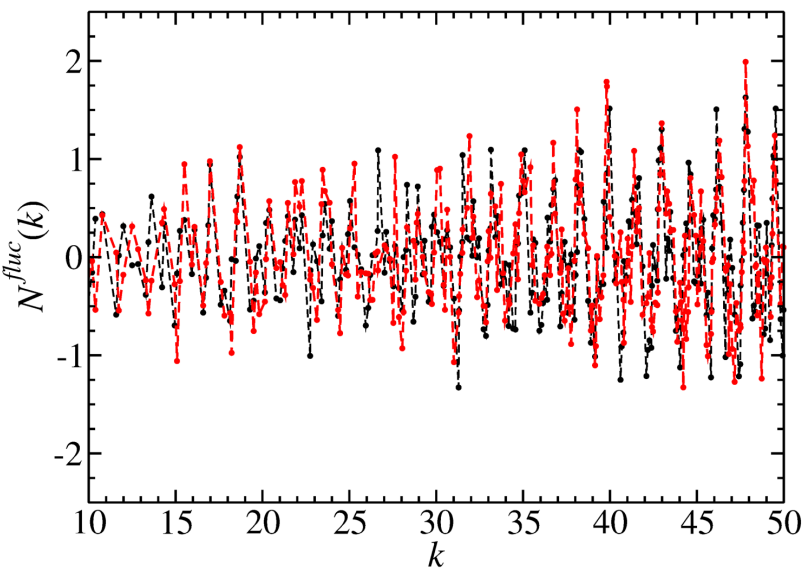

Fig. 11. The same as Fig. 2 for the elliptical NB.

These differences arise due to the chirality property which leads to the cancellation of periodic orbits with an odd number of reflections and the additional spin degree of freedom which gives rise to the additional phase factor. In Figs. 9 and 10 we compare the fluctuating part of the integrated spectral density obtained from 


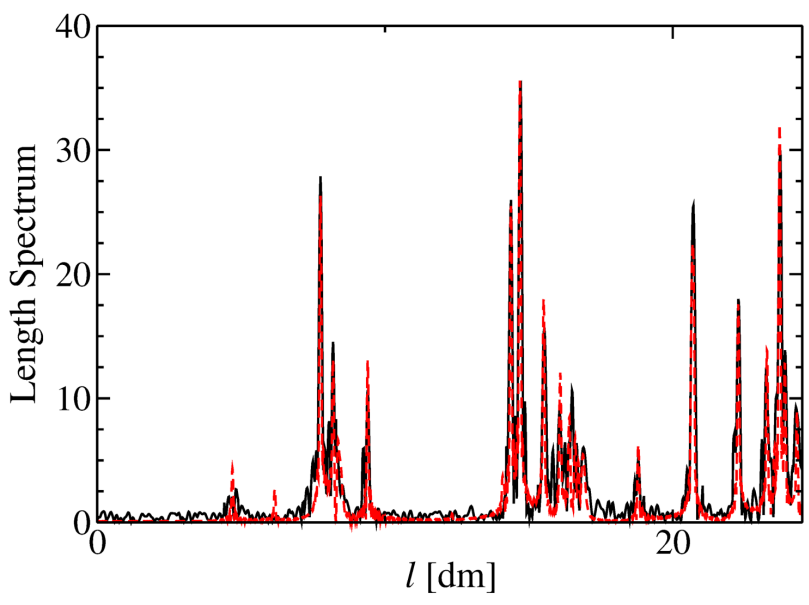

Fig. 12. The same as Fig. 3 for the elliptical NB.

5000 eigenvalues of the circular NB, respectively, 1000 eigenvalues of the elliptical NB with those deduced from the trace formula Eq. (75) using the entries of the trace formulae Eqs. (3) and (19) for the corresponding QB. Figures 11 and 12 show the results for the elliptical NB, for which we computed about 2000 eigenvalues. For the circular NB the trace formula is identical with the one which was derived in [35] for circular graphene billiards in the energy range, where they exhibit relativistic phenomena.

\section{Conclusions}

In the first part of this article we present a quantization condition for elliptical NBs in terms of the Mathieu functions resulting from the boundary conditions. In the second part we introduce a trace formula which should be applicable to NBs with a convex and smooth boundary. We tested it for the circular and the elliptical NBs which both exhibit spectral properties following Poissonian statistics. The aim is the understanding of the spectral properties of NBs for cases where they deviate from those expected according to the BGS or the BT conjecture depending on whether the billiard shape generates a chaotic or regular classical dynamics, as would be expected, e.g., from the results obtained for sectorshaped graphene billiards [64].

\section{Acknowledgments}

This work was initiated during my stay at the TU Darmstadt and supported at that time by the Deutsche Forschungsgemeinschaft (DFG) within the Collaborative Research Center 643. Furthermore it was supported by NNSF of China under Grant Nos. 11775100 and 11961131009. I would like to thank Remy Dubertrand and Martin Sieber for fruitful discussions.

\section{Appendix A: Some properties of the Mathieu functions}

Using elliptic coordinates

$x(\mu, \phi)=\cosh \mu \cos \phi$

$y(\mu, \phi)=\sinh \mu \sin \phi$

where

$$
\phi \in[0,2 \pi[, \quad \mu \in[0, \infty[
$$

and defining $z=\mathrm{e}^{\mu} \mathrm{e}^{\mathrm{i} \phi}, w(z)=x(\mu, \phi)+\mathrm{i} y(\mu, \phi)$ becomes

$$
\begin{aligned}
& w(z)=\frac{1}{2}\left(\mathrm{e}^{\mu} \mathrm{e}^{\mathrm{i} \phi}+\mathrm{e}^{-\mu} \mathrm{e}^{-\mathrm{i} \phi}\right), \\
& |w(z)|=\sqrt{x^{2}+y^{2}}=\sqrt{\cosh ^{2} \mu_{0}-\sin ^{2} \phi}, \\
& z w^{\prime}(z)=\frac{1}{2}\left(\mathrm{e}^{\mu} \mathrm{e}^{\mathrm{i} \phi}-\mathrm{e}^{-\mu} \mathrm{e}^{-\mathrm{i} \phi}\right), \\
& \left|z w^{\prime}(z)\right|^{2}=\left[\cosh ^{2} \mu-\cos ^{2} \phi\right] .
\end{aligned}
$$

The boundary of an ellipse with minor axis $\sinh \mu_{0}$ and major axis $\cosh \mu_{0}$ is parametrized by $\phi$ :

With

$$
|w(\phi)|=\sqrt{\cosh ^{2} \mu_{0}-\sin ^{2} \phi} .
$$

$$
\frac{\partial}{\partial x}+\mathrm{i} \frac{\partial}{\partial y}=\frac{1}{z^{\star}\left[w^{\prime}(z)\right]^{\star}}\left(\frac{\partial}{\partial \mu}+\mathrm{i} \frac{\partial}{\partial \phi}\right)
$$

the Dirac equation reads in elliptic coordinates

$$
\begin{aligned}
& k \psi= \\
& \left(\begin{array}{cc}
0 & \frac{-\mathrm{i}}{z\left[w^{\prime}(z)\right]}\left(\frac{\partial}{\partial \mu}-\mathrm{i} \frac{\partial}{\partial \phi}\right) \\
\frac{-\mathrm{i}}{z^{\star}\left[w^{\prime}(z)\right]^{\star}}\left(\frac{\partial}{\partial \mu}+\mathrm{i} \frac{\partial}{\partial \phi}\right) & 0
\end{array}\right) \boldsymbol{\psi} .
\end{aligned}
$$

The Schrödinger equation describing the corresponding quantum billiard is obtained by applying the Dirac operator twice, yielding with

$$
\begin{gathered}
\frac{\partial^{2}}{\partial x^{2}}+\frac{\partial^{2}}{\partial y^{2}}=\left(\frac{\partial}{\partial x}+\mathrm{i} \frac{\partial}{\partial y}\right)\left(\frac{\partial}{\partial x}-\mathrm{i} \frac{\partial}{\partial y}\right)= \\
\frac{1}{2\left|z w^{\prime}(z)\right|^{2}}\left(\frac{\partial^{2}}{\partial \mu^{2}}+\frac{\partial^{2}}{\partial \phi^{2}}\right)
\end{gathered}
$$

two separate equations for $\phi$ and $\mu$, which, in distinction to the case of the circular billiard are linked by a common constant $h=h(k)$ which depends on $k$ :

$$
\begin{aligned}
& \frac{\partial^{2}}{\partial \phi^{2}} \Phi(\phi)+\left(h(k)-k^{2} \cos ^{2} \phi\right) \Phi(\phi)=0, \\
& -\frac{\partial^{2}}{\partial \mu^{2}} M(\mu)+\left[h(k)-k^{2} \cosh ^{2} \mu\right] M(\mu)=0 .
\end{aligned}
$$

The solutions $\psi(\mu, \phi)$ are given as an expansion in terms of the Mathieu functions

$$
\psi(\mu, \phi)=\sum_{n} a_{n} M_{n}(\mu) \Phi_{n}(\phi) .
$$

The periodic Mathieu functions $\Phi_{n}(\phi)$ may be classified according to their reflection symmetry with respect to the major and minor axes of the elliptical billiard 


$$
\begin{aligned}
& c e_{2 n}(\phi, k)=\sum_{m=0}^{\infty} A_{2 m}^{2 n}(k) \cos [2 m \phi], \\
& c e_{2 n+1}(\phi, k)=\sum_{m=0}^{\infty} A_{2 m+1}^{2 n+1}(k) \cos [(2 m+1) \phi], \\
& s e_{2 n}(\phi, k)=\sum_{m=0}^{\infty} B_{2 m}^{2 n}(k) \sin [2 m \phi], \quad B_{0}^{2 n}=0, \\
& s e_{2 n+1}(\phi, k)=\sum_{m=0}^{\infty} B_{2 m+1}^{2 n+1}(k) \sin [(2 m+1) \phi],
\end{aligned}
$$

where the coefficients $A_{m}^{n}$ and $B_{m}^{n}$ are functions of $k$. Furthermore, with $\delta_{n m}$ denoting the Kronecker $\delta$ :

$$
\begin{aligned}
& \frac{1}{\pi} \int_{0}^{2 \pi} c e_{n}(\phi, k) c e_{m}(\phi, k) \mathrm{d} \phi=\delta_{n m}, \\
& \frac{1}{\pi} \int_{0}^{2 \pi} s e_{n}(\phi, k) s e_{m}(\phi, k) \mathrm{d} \phi=\delta_{n m}, \\
& \frac{1}{\pi} \int_{0}^{2 \pi} c e_{n}(\phi, k) s e_{m}(\phi, k) \mathrm{d} \phi=0,
\end{aligned}
$$

and the normalization is chosen as

$$
\begin{aligned}
& 2\left[A_{0}^{2 n}\right]^{2}+\sum_{m=1}^{\infty}\left[A_{2 n}^{2 m}\right]^{2}=1, \quad \sum_{m=0}^{\infty}\left[A_{2 m+1}^{2 n+1}\right]^{2}=1 \\
& \sum_{m=0}^{\infty}\left[B_{2 m+1}^{2 n+1}\right]^{2}=\sum_{m=1}^{\infty}\left[B_{2 m}^{2 n}\right]^{2}=1 \\
& \sum_{n=0}^{\infty} A_{2 l+1}^{2 n+1} A_{2 m+1}^{2 n+1}=\sum_{n=0}^{\infty} A_{2 l}^{2 n} A_{2 m}^{2 n}=\delta_{l m} \\
& \sum_{n=0}^{\infty} A_{0}^{2 n} A_{2 m}^{2 n}=\frac{1}{2} \delta_{0 m}, \\
& \sum_{n=0}^{\infty} B_{2 l+1}^{2 n+1} B_{2 m+1}^{2 n+1}=\sum_{n=1}^{\infty} B_{2 l}^{2 n} B_{2 m}^{2 n}=\delta_{l m} .
\end{aligned}
$$

The associated radial Mathieu function $M_{n}\left(\mu_{0}\right)$ are given as

$$
\begin{aligned}
& C e_{2 n}(\mu, k)=\frac{p_{2 n}}{c e_{2 n}\left(\frac{\pi}{2}, k\right)} \sum_{m=0}^{\infty} A_{2 m}^{2 n}(k) J_{2 m}(2 k \sinh \mu), \\
& C e_{2 n+1}(\mu, k)=-\frac{p_{2 n+1}}{c e_{2 n+1}^{\prime}\left(\frac{\pi}{2}, k\right)} \operatorname{coth} \mu \\
& \quad \times \sum_{m=0}^{\infty}(2 m+1) A_{2 m+1}^{2 n+1}(k) J_{2 m+1}(2 k \sinh \mu), \\
& S e_{2 n}(\mu, k)=\frac{s_{2 n}}{s e_{2 n}^{\prime}\left(\frac{\pi}{2}, k\right)} \operatorname{coth} \mu \\
& \quad \times \sum_{m=1}^{\infty} 2 m B_{2 m}^{2 n}(k) J_{2 m}(2 k \sinh \mu),
\end{aligned}
$$

$$
\begin{aligned}
& S e_{2 n+1}(\mu, k)=-\frac{s_{2 n+1}}{s e_{2 n+1}^{\prime}\left(\frac{\pi}{2}, k\right)} \\
& \quad \times \sum_{m=0}^{\infty} B_{2 m+1}^{2 n+1}(k) J_{2 m+1}(2 k \sinh \mu),
\end{aligned}
$$

with the notations

$$
\begin{aligned}
& p_{2 n}=\frac{c e_{2 n}(0, k) c e_{2 n}\left(\frac{\pi}{2}, k\right)}{A_{0}^{2 n}(k)}, \\
& p_{2 n+1}=-\frac{c e_{2 n+1}(0, k) c e_{2 n+1}^{\prime}\left(\frac{\pi}{2}, k\right)}{k A_{1}^{2 n+1}(k)}, \\
& s_{2 n}=\frac{s e_{2 n}^{\prime}(0, k) s e_{2 n}^{\prime}\left(\frac{\pi}{2}, k\right)}{k^{2} B_{2}^{2 n}(k)}, \\
& s_{2 n+1}=-\frac{s e_{2 n+1}^{\prime}(0, k) s e_{2 n+1}\left(\frac{\pi}{2}, k\right)}{k B_{1}^{2 n+1}(k)} .
\end{aligned}
$$

The solutions of the Dirac equation of the corresponding $\mathrm{NB}$ can be written in the form

$$
\left(\begin{array}{l}
\psi_{1} \\
\psi_{2}
\end{array}\right)=\left(\begin{array}{c}
\sum_{l} a_{l} \mathrm{i}^{l} J_{l}(k|w(z)|) \mathrm{e}^{\mathrm{i} l \theta(z)} \\
\sum_{l} b_{l} \mathrm{i}^{l} J_{l}(k|w(z)|) \mathrm{e}^{\mathrm{i} l \theta(z)}
\end{array}\right),
$$

with $\mathrm{e}^{\mathrm{i} \theta(z)}=\frac{w(z)}{|w(z)|}$ and $|w(z)|, w(z)$ given in Eqs. (A7) and (31). Inserting this ansatz into Eq. (A9) yields with

$$
\left(\frac{\partial}{\partial \mu}+\mathrm{i} \frac{\partial}{\partial \phi}\right)|w(z)|=z^{\star}\left[w^{\prime}(z)\right]^{\star} \mathrm{e}^{\mathrm{i} \theta}
$$

and

$$
\begin{aligned}
& \left(\frac{\partial}{\partial \mu}+\mathrm{i} \frac{\partial}{\partial \phi}\right) \mathrm{e}^{\mathrm{i} l \theta}=-l \frac{z^{\star}\left[w^{\prime}(z)\right]^{\star}}{|w(z)|} \mathrm{e}^{\mathrm{i}(l+1) \theta}, \\
& \left(\frac{\partial}{\partial \mu}-\mathrm{i} \frac{\partial}{\partial \phi}\right) \mathrm{e}^{\mathrm{i} l \theta}=l \frac{z\left[w^{\prime}(z)\right]}{|w(z)|} \mathrm{e}^{\mathrm{i}(l-1) \theta},
\end{aligned}
$$

as condition for it to be a solution of the Dirac equation Eq. (A9):

$\sum_{l} a_{l} \mathrm{i}^{l} J_{l}(k|w(z)|) \mathrm{e}^{\mathrm{i} l \theta}=\sum_{l} b_{l} \mathrm{i}^{l-1} J_{l-1}(k|w(z)|) \mathrm{e}^{\mathrm{i}(l-1) \theta}$,

$\sum_{l} b_{l} \mathrm{i}^{l} J_{l}(k|w(z)|) \mathrm{e}^{\mathrm{i} l \theta}=\sum_{l} a_{l} \mathrm{i}^{l+1} J_{l+1}(k|w(z)|) \mathrm{e}^{\mathrm{i}(l+1) \theta}$.

These equations can be expanded in terms of the Mathieu functions

$\mathrm{i}^{2 l} J_{2 l}(k|w(z)|) \cos (2 l \theta)=\sum_{n=0}^{\infty} \frac{A_{2 l}^{2 n}(k)}{p_{2 n}} C e_{2 n}(\mu) c e_{2 n}(\phi)$,

$$
\begin{aligned}
& \mathrm{i}^{2 l+1} J_{2 l+1}(k|w(z)|) \cos ((2 l+1) \theta)= \\
& -\mathrm{i} \sum_{n=0}^{\infty} \frac{A_{2 l+1}^{2 n+1}(k)}{p_{2 n+1}} C e_{2 n+1}(\mu) c e_{2 n+1}(\phi), \\
& \mathrm{i}^{2 l} J_{2 l}(k|w(z)|) \sin [2 l \theta]=\sum_{n=0}^{\infty} \frac{B_{2 l}^{2 n}(k)}{s_{2 n}} S_{2 n}(\mu) s e_{2 n}(\phi),
\end{aligned}
$$




$$
\begin{aligned}
& \mathrm{i}^{2 l+1} J_{2 l+1}(k|w(z)|) \sin [(2 l+1) \theta]= \\
& \quad \mathrm{i} \sum_{n=0}^{\infty} \frac{B_{2 l+1}^{2 n+1}(k)}{s_{2 n+1}} S e_{2 n+1}(\mu) s e_{2 n+1}(\phi) .
\end{aligned}
$$

With the notation

$$
\begin{aligned}
& \tilde{A}_{n=2 \nu+\sigma, l=2 \lambda+\sigma}=\mathrm{i}^{-\sigma} \frac{A_{2 \lambda+\sigma}^{2 \nu+\sigma}}{p_{2 \nu+\sigma}}, \\
& \tilde{B}_{n=2 \nu+\sigma, l=2 \lambda+\sigma}=\mathrm{i}^{\sigma} \frac{B_{2 \lambda+\sigma}^{2 \nu+\sigma}}{s_{2 \nu+\sigma}},
\end{aligned}
$$

where $\sigma=0,1$ we obtain for Eq. (A20):

$$
\begin{aligned}
& \sum_{l} a_{l} \sum_{n=0}^{\infty}\left\{\tilde{A}_{n l} C e_{n}(\mu) c e_{n}(\phi)+\mathrm{i} \tilde{B}_{n l} S e_{n}(\mu) s e_{n}(\phi)\right\}= \\
& \sum_{l} b_{l+1} \sum_{n=0}^{\infty}\left\{\tilde{A} n l C e_{n}(\mu) c e_{n}(\phi)+\mathrm{i} \tilde{B}_{n l} S e_{n}(\mu) s e_{n}(\phi)\right\},
\end{aligned}
$$

$$
\begin{aligned}
& \sum_{l} b_{l} \sum_{n=0}^{\infty}\left\{\tilde{A}_{n l} C e_{n}(\mu) c e_{n}(\phi)+\mathrm{i} \tilde{B}_{n l} S e_{n}(\mu) s e_{n}(\phi)\right\}= \\
& \sum_{l} a_{l-1} \sum_{n=0}^{\infty}\left\{\tilde{A}_{n l} C e_{n}(\mu) c e_{n}(\phi)+\mathrm{i} \tilde{B}_{n l} S e_{n}(\mu) s e_{n}(\phi)\right\} .
\end{aligned}
$$

Multiplying these equations with $s e_{m}(\phi)$ or $c e_{m}(\phi)$ and integrating over $\phi$ yields with the orthogonality relations Eq. (A14):

$$
\begin{aligned}
& \sum_{l} a_{l} A_{m l} C e_{m}(\mu)=\sum_{l} b_{l+1} A_{m l} C e_{m}(\mu), \\
& \sum_{l} a_{l} B_{m l} S e_{m}(\mu)=\sum_{l} b_{l+1} B_{m l} S e_{m}(\mu), \\
& \sum_{l} b_{l} A_{m l} C e_{m}(\mu)=\sum_{l} a_{l-1} A_{m l} C e_{m}(\mu), \\
& \sum_{l} b_{l} B_{m l} S e_{m}(\mu)=\sum_{l} a_{l-1} B_{m l} S e_{m}(\mu) .
\end{aligned}
$$

Next we divide these equations by the Mathieu functions $C e_{m}(\mu)$, respectively, $S e_{m}(\mu)$, then multiply them with the coefficients $A_{m l^{\prime}}$, respectively, $B_{m l^{\prime}}$ and employ the orthogonality relation Eq. (A15) yielding

$$
a_{l}=b_{l+1}, \quad b_{l}=-a_{l-1}
$$

and thus in both cases $b_{l}=a_{l-1}$.

\section{References}

[1] M.V. Berry, Eur. J. Phys. 2, 91 (1981).

[2] Y.G. Sinai, Russ. Math. Surv. 25, 137 (1970).

[3] V.F. Lazutkin, Math. USSR Izv. 7, 185 (1973).

[4] L.A. Bunimovich, Commun. Math. Phys. 65, 295 (1979).

[5] Chaos and Quantum Physics, Eds. M.J. Giannoni, A. Voros, J. Zinn-Justin, Les Houches, Vol. 52, Elsevier, Amsterdam 1989.
[6] H.J. Stöckmann, Quantum Chaos: An Introduction, Cambridge University Press, Cambridge 1990.

[7] A. Richter, in: Emerging Applications of Number Theory, The IMA Volumes in Mathematics and Its Applications, Eds. D.A. Hejhal, J. Friedmann, M.C. Gutzwiller, A.M. Odlyzko, Springer, New York 1999, p. 479.

[8] F. Haake, Quantum Signatures of Chaos, SpringerVerlag, Heidelberg 2001.

[9] H.J. Stöckmann, J. Stein, Phys. Rev. Lett. 64, 2215 (1990).

[10] S. Sridhar, Phys. Rev. Lett. 67, 785 (1991).

[11] J. Stein, H.-J. Stöckmann, Phys. Rev. Lett. 68, 2867 (1992).

[12] H.D. Gräf, H.L. Harney, H. Lengeler, C.H. Lewenkopf, C. Rangacharyulu, A. Richter, P. Schardt, H.A. Weidenmüller, Phys. Rev. Lett. 69, 1296 (1992).

[13] P. So, S. Anlage, E. Ott, R. Oerter, Phys. Rev. Lett. 74, 2662 (1995).

[14] S. Deus, P. Koch, L. Sirko, Phys. Rev. E 52, 1146 (1995).

[15] B. Dietz, A. Richter, Chaos 25, 097601 (2015).

[16] M.V. Berry, J. Phys. A 10, 2083 (1977).

[17] M.V. Berry, in: Structural Stability in Physics, Eds. N. Guttinger, H. Eikemeier, Berlin 1979.

[18] G. Casati, F. Valz-Gris, I. Guarnieri, Lett. Nuovo Cimento 28, 279 (1980).

[19] O. Bohigas, M.J. Giannoni, C. Schmit, Phys. Rev. Lett. 52, 1 (1984).

[20] M.V. Berry, M. Tabor, J. Phys. A 10, 371 (1977).

[21] F. Leyvraz, C. Schmit, T.H. Seligman, J. Phys. A 29, L575 (1996).

[22] C. Dembowski, H.D. Gräf, A. Heine, H. Rehfeld, A. Richter, C. Schmit, Phys. Rev. E 62, R4516 (2000).

[23] C. Dembowski, B. Dietz, H.-D. Gräf, A. Heine, F. Leyvraz, M. Miski-Oglu, A. Richter, T.H. Seligman, Phys. Rev. Lett. 90, 014102 (2003).

[24] O. Knill, Elemente Math. 53, 89 (1998).

[25] B. Gutkin, J. Phys. A 40, F761 (2007).

[26] B. Dietz, T. Guhr, B. Gutkin, M. Miski-Oglu, A. Richter, Phys. Rev. E 90, 022903 (2014).

[27] M.V. Berry, R.J. Mondragon, Proc. R. Soc. Lond. A 412, 53 (1987).

[28] K.S. Novoselov, A.K. Geim, S.V. Morozov, D. Jiang, Y. Zhang, S. Dubonos, I. Grigorieva, A. Firsov, Science 306, 666 (2004).

[29] A.H. Castro Neto, F. Guinea, N.M.R. Peres, K.S. Novoselov, A.K. Geim, Rev. Mod. Phys. 81, 109 (2009).

[30] C.W.J. Beenakker, Rev. Mod. Phys. 80, 1337 (2008).

[31] P.G. Silvestrov, K.B. Efetov, Phys. Rev. Lett. 98 , 016802 (2007).

[32] F. Libisch, C. Stampfer, J. Burgdörfer, Phys. Rev. B 79, 115423 (2009) 
[33] J. Wurm, A. Rycerz, İ. Adagideli, M. Wimmer, K. Richter, H.U. Baranger, Phys. Rev. Lett. 102, 056806 (2009).

[34] L. Huang, Y.C. Lai, C. Grebogi, Phys. Rev. E 81 , 055203 (2010).

[35] J. Wurm, K. Richter, İ. Adagideli, Phys. Rev. B 84 075468 (2011).

[36] B. Dietz, T. Klaus, M. Miski-Oglu, A. Richter, Phys Rev. B 91, 035411 (2015).

[37] B. Dietz, T. Klaus, M. Miski-Oglu, A. Richter, M. Wunderle, C. Bouazza, Phys. Rev. Lett. 116 023901 (2016).

[38] M. Gutzwiller, Chaos in Classical and Quantum Mechanics, Springer Verlag, New York 1990.

[39] M.C. Gutzwiller, J. Math. Phys. (New York) 12, 343 (1971).

[40] A. Einstein, Verh. Dtsch. Phys. Ges. 19, 82 (1917).

[41] L. Brillouin, J. Phys. Radium 7, 353 (1926).

[42] J.B. Keller, Ann. Phys. 4, 180 (1958).

[43] M.V. Berry, J.P. Keating, J. Phys. A 23, 4839 (1990).

[44] M. Sieber, F. Steiner, Phys. Rev. Lett. 67, 1941 (1991).

[45] E.B. Bogomolny, Nonlinearity 5, 805 (1992).

[46] T. Harayama, A. Shudo, Phys. Lett. A 165, 417 (1992).

[47] M. Sieber, N. Pavloff, C. Schmit, Phys. Rev. E 55 2279 (1997)

[48] M. Sieber, Nonlinearity 11, 1607 (1998).

[49] J. Bolte, S. Keppeler, Ann. Phys. 274, 125 (1999).
[50] S. Keppeler, Ann. Phys. 304, 40 (2003).

[51] P. Carmier, D. Ullmo, Phys. Rev. B 77, 245413 (2008).

[52] S.C. Creagh, R.G. Littlejohn, Phys. Rev. A 44, 836 (1991).

[53] S.M. Reimann, M. Brack, A.G. Magner, J. Blaschke, M.V.N. Murthy, Phys. Rev. A 53, 39 (1996).

[54] M. Sieber, J. Phys. A 30, 4563 (1997).

[55] M.L. Mehta, Random Matrices, Academic Press, London 1990.

[56] H. Weyl, J. Reine Angew. Math. 141, 1 (1912).

[57] N.W. MacLachlan, Theory and Application of Mathieu Functions, Oxford University Press, London 1947.

[58] P.M. Morse, H. Feshbach, Methods of Theoretical Physics, McGraw-Hill, New York 1953.

[59] M. Abramowitz, I.A. Stegun, Handbook of Mathematical Functions with Formulas, Graphs and Mathematical Tables, Dover Publications, New York 2013.

[60] B. Dietz, U. Smilansky, Chaos 3, 581 (1993).

[61] H. Waalkens, J. Wierig, H.R. Dullin, Ann. Phys. 260, 50 (1997)

[62] P. Yu, B. Dietz, L. Huang, New J. Phys. 21, 073039 (2019).

[63] A. Bäcker, Springer Lecture Notes in Physics 618, 91 (2003).

[64] P. Yu, Z.Y. Li, H.Y. Xu, L. Huang, B. Dietz, C. Grebogi, Y.C. Lai, Phys. Rev. E 94, 062214 (2016). 